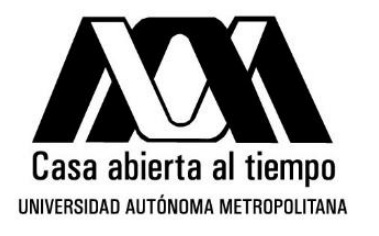

Universidad Autónoma Metropolitana

Unidad Iztapalapa

División de Ciencias Básicas e Ingeniería

\title{
ANÁlisis Y APLiCACIÓN DE JUEgOS NO CoOperativos con Estructura Supermodular
}

\author{
Tesis que presenta: \\ Julieta Del Rosario Sevilla Brambila \\ Para obtener el grado de: \\ Maestra en Ciencias Matemáticas Aplicadas e \\ INDUSTRIALES
}

Asesor: Dr. José Raúl Montes de Oca Machorro

Jurado calificador:

Presidente: Dr. Julio César García Corte

Secretario: Dr. José Raúl Montes de Oca Machorro

Vocal: Dr. Víctor Hugo Vázquez Guevara

Ciudad de México. 13 de Diciembre, 2017 





\section{Agradecimientos}

A Faviola por aceptar que no quería volver a casa y no intentar detenerme. A papá, porque hasta la fecha me sigue alentando, al menos en sueños, a terminar lo que empiezo. A Katya, Cinthia y Karlangas, por su apoyo moral, aunque no se enteraran ni de lo que estaba haciendo, con especial mención a la última por siempre distraerme de mis problemas, contándome otros.

A la Universidad Autónoma Metropolitana por abrirme sus puertas, sobre todo a la MCMAI, por aceptarme aún después de llegar tarde al examen de admisión.

Al CONACYT por apoyarme económicamente durante este posgrado.

Al profesor Patricio, por hacer de su curso un hogar y brindarme 4 horas de bienestar a la semana; a Arisbeth, por mostrarme que no estoy peleada con las actividades artísticas, sin ellos no hubiera soportado ni el primer trimestre.

Al Dr. Montes de Oca, por su guía, paciencia, por animarme a seguir mi corazonada con el rumbo que quería darle a esta tesis y sobre todo, por no dejarme desistir de ella.

A la Dra. Saavedra y al Dr. Núñez, por brindarme palabras de apoyo en momentos de adversidad y alentarme a creer en mis capacidades.

A los amigos que llegaron y a los que se quedaron a otra tesis, no me bastan las palabras para mostrar mi gratitud al subconjunto que sigue conmigo, brindándome su apoyo y oido. 



\section{Índice general}

$\begin{array}{ll}\text { Notación } & \text { IX }\end{array}$

$\begin{array}{ll}\text { Introducción } & 11\end{array}$

1. Preliminares 15

1.1. Introducción . . . . . . . . . . . . . . . . . 15

1.2. Latices . . . . . . . . . . . . . . . . . . . . 15

1.2.1. Conjuntos parcialmente ordenados . . . . . . . . . 15

1.2.2. Latices, sublatices y propiedades . . . . . . . . . . . 17

1.2.3. Orden inducido de un conjunto . . . . . . . . . . . . . 21

1.2.4. Puntos fijos . . . . . . . . . . . . . . . . 23

1.3. Funciones supermodulares _. . . . . . . . . . . . . . 25

1.3.1. Conjunto de soluciones óptimas _ . . . . . . . . . . . 28

1.4. Conclusiones . . . . . . . . . . . . . . . . . . . 30

2. Juegos Supermodulares no Cooperativos 31

2.1. Introducción . . . . . . . . . . . . . . . . . . . 31

2.2. Definición de juego supermodular . . . . . . . . . . . . . 32

2.3. Existencia de puntos de equilibrio . . . . . . . . . . . . . 34

2.4. Algoritmos . . . . . . . . . . . . . . . . . . 36

2.4.1. Optimización Round-Robin . . . . . . . . . . . . 36

2.4.2. Optimización simultánea . . . . . . . . . . . . . 37

2.5. Conclusiones . . . . . . . . . . . . . . . . . . 38

3. Aplicaciones 39

3.1. Introducción . . . . . . . . . . . . . . . . . . . 39

3.2. Antecedentes . . . . . . . . . . . . . . . . . . . . . 39

3.2.1. Producción con bienes complementarios [10] . . . . . . . . . 39

3.2.2. Redes AdHoc inalámbricas [5] . . . . . . . . . . . . . . 41 
3.2.3. Fijación de precios con productos reemplazables [1], [10] . . . 43

3.2.4. Oligopolios multimercado [10], [12] . . . . . . . . . . . . . . . 44

3.2.5. Teoría de Colas [13] . . . . . . . . . . . . . . . . . . 44

3.2.6. Búsqueda de socios comerciales $[1],[10] \ldots$. . . . . . . . 45

3.3. Productos parcialmente complementarios . . . . . . . . . . . 46

3.3.1. Planteamiento del modelo . . . . . . . . . . . . . . . . . . . 47

3.3.2. Demandas distribuidas uniformemente . . . . . . . . . . . 48

3.3.3. Ejemplos . . . . . . . . . . . . . . . . . . . . 49

3.3.4. Comentarios sobre demandas distribuidas exponencialmente . 62

3.4. Conclusiones . . . . . . . . . . . . . . . . . 66

4. Conclusiones y perspectivas $\quad 67$

$\begin{array}{ll}\text { A. Algunas demostraciones } & 69\end{array}$

B. Función de utilidad esperada $\quad 74$

B.1. Demandas distribuidas uniformemente . . . . . . . . . . . . . . . 75

B.1.1. Diferencias crecientes ............. 77

$\begin{array}{ll}\text { C. Códigos } & 81\end{array}$

$\begin{array}{ll}\text { Bibliografía } & 85\end{array}$ 


\section{Notación}

$\begin{array}{ll}x \vee y & \text { Mínima cota superior de } x \text { y } y \\ x \wedge y & \text { Máxima cota inferior de } x \text { y } y \\ \mathscr{L}(X) & \text { Conjunto de sublatices no vacías de } X \\ S_{t} & \text { Sección de } S \text { sobre } t \\ \Pi_{T} S & \text { Proyección de } S \text { sobre } T \\ \operatorname{argmáx} x_{x \in X} f(x) & \text { Conjunto de soluciones óptimas de } f \\ x_{-i} & \text { Perfil de estrategias correspondiente a todos los jugadores menos } \\ & \text { el } i \text {-ésimo } \\ \left(\mathbf{x} ; y_{i}\right) & \text { Perfil de estrategias con la correspondiente al jugador } i \text { cambiada } \\ S_{i}\left(x_{-i}\right) & \text { por } y_{i} \\ S_{-i} & \text { Estrategias admisibles para el jugador } i \text { dado } x_{-i} \\ R_{i} & \text { Proyección del conjunto de estrategias admisibles en las estrategias } \\ Y_{i}\left(x_{-i}\right) & \text { de todos los jugadores menos el } i \text {-ésimo } \\ Y(\mathbf{x}) & \text { Función de utilidad del } i \text {-ésimo jugador } \\ \mathbb{1}_{A} & \text { Correspondencia de mejor respuesta del jugador } i \\ & \text { Correspondencia de mejor respuesta conjunta }\end{array}$





\section{Introducción}

Esta tesis pertenece al área de teoría de juegos [6], [7], [14]. Dicha teoría estudia problemas de toma de decisiones multipersonales. Este tipo de problemas aparecen comunmente en diversas áreas, como son economía e investigación de operaciones [1], [2], [5], [7], [10].

La tesis se enfoca en los juegos supermodulares no cooperativos. El interés en la no cooperatividad se debe a que es razonable suponer que los jugadores no se pueden comunicar o no pueden colaborar entre ellos. Además, en los juegos no cooperativos se pueden caracterizar los equilibrios de Nash como puntos fijos de una correspondencia dependiente de las funciones de utilidad [6].

Por otro lado, la propiedad de supermodularidad en juegos no cooperativos, permite aproximar equilibrios mediante algoritmos, sin usar teoremas de punto fijo tipo Brouwer, Kakutani, etc. [15], esto hace más accesible la búsqueda de soluciones en dichos juegos [10].

El objetivo general de la tesis es mostrar la aplicabilidad de los juegos supermodulares no cooperativos y las ventajas que estos brindan al proveer resultados que no solo aseguran la existencia de equilibrios de Nash sino que, también cuentan con algoritmos para aproximar los equilibrios mencionados de manera explícita.

Cabe mencionar que, los juegos supermodulares no cooperativos han sido usados en diversas ramas, como son: teoría de colas [4], [13], administración de recursos [10], economía [10], redes [5], teoría de gráficas [10], entre otras.

En este trabajo, además de algunas de las aplicaciones mencionadas, se estudia el modelo de productos parcialmente complementarios con demanda aleatoria [2], [3]. El interés en este modelo se debe a que, las aplicaciones citadas en el párrafo anterior son deterministas y los productos parcialmente complementarios tienen un enfoque 
estocástico, situación que hasta el momento no se ha localizado en la literatura especializada.

Además, es importante mencionar que, como aportación de este trabajo se analizan dichos modelos usando las bondades que provee la supermodularidad en los juegos no cooperativos. Particularmente, se muestra que, para demandas con distribuciones uniformes y exponenciales, con ciertas propiedades, el modelo puede verse como un juego supermodular no cooperativo, lo cual permite aproximar equilibrios en casos concretos, usando los algoritmos de optimización Round-Robin y optimización simultánea; en contraste con el artículo de X. Fang [2], donde de plantea que el equilibrio puede ser encontrado resolviendo un sistema de ecuaciones diferenciales.

La estructura de la tesis es la siguiente:

En el capítulo 1, se presentan los conceptos de latiz y función supermodular, además, algunas propiedades de estos que se usan para caracterizar los conjuntos de estrategias admisibles y las funciones de utilidad en los juegos supermodulares no cooperativos.

En el capítulo 2, se dan los conceptos básicos de teoría de juegos que son necesarios para la comprensión del tipo de juegos que se abordan en esta tesis; seguido de esto, se definirán los juegos supermodulares no cooperativos, los algoritmos que permiten aproximar equilibrios en estos y algunos resultados que aseguran la existencia de dichos equilibrios, cuando los perfiles de estrategias y las funciones de utilidad cumplen ciertas propiedades.

En el capítulo 3, se muestran modelos en los cuales se han utilizado los juegos supermodulares no cooperativos, haciendo énfasis en el modelo de productos parcialmente complementarios con demanda aleatoria. Este modelo, fue planteado como un juego no cooperativo [2] y en este capítulo se prueba que, para algunas demandas, las funciones de utilidad del modelo cumplen las hipótesis de diferencias crecientes y supermodularidad, de esto que dicho modelo se pueda ver como un juego supermodular no cooperativo y se aproximan equilibrios para algunos ejemplos concretos.

En el apéndice A, se dan algunas demostraciones de resultados de los capítulos 1 y 2. En el apéndice B, se muestra otra presentación de la función de utilidad esperada planteada en [2] y la forma que esta tiene cuando las demandas se distribuyen uniformemente, seguido de esto, se exhibe que dicha función tiene diferencias crecientes y 
se concluye la supermodularidad de esta. En el apéndice C, se muestran el programa elaborado en MatLab para el algoritmo de optimización Round-Robin y otro código realizado en Mathematica para determinar la función de utilidad esperada cuando las demandas se distribuyen uniformemente. 



\section{Capítulo 1}

\section{Preliminares}

\subsection{Introducción}

El objetivo de este capítulo es presentar los conceptos de latiz y función supermodular, así como algunas propiedades de estos, que son de suma importancia en el desarrollo de los juegos supermodulares.

Inicialmente, se darán conceptos como relación binaria, conjunto parcialmente ordenado, entre otros, que son indispensables para poder definir una latiz, además se proveerán algunos ejemplos y atributos de dichos conjuntos.

Teniendo la definición de latiz se dará la de función supermodular y algunas propiedades. Se concluirá el capítulo dando caracterizaciones de las funciones supermodulares en términos de la propiedad de diferencias crecientes y definiendo el conjunto de soluciones óptimas de una función y cualidades de este.

Los conceptos de este capítulo fueron revisados en [1], [10] y [11].

\subsection{Latices}

\subsubsection{Conjuntos parcialmente ordenados}

En este apartado se evocará lo que es un conjunto parcialmente ordenado y algunas cotas en estos conjuntos. Estos conceptos serán de utilidad para poder definir una latiz. 
Primeramente, se dará el concepto de relación binaria, después, se definirá lo que es un conjunto parcialmente ordenado y se darán algunos ejemplos para afianzar dichas definiciones.

Definición 1.1. Se dice que $\preceq$ es una relación binaria sobre un conjunto $X$, si para cualesquiera $x, y \in X$ se tiene que $x \preceq y$ es verdadero o $x \preceq y$ es falso.

Definición 1.2. $X$ es un conjunto parcialmente ordenado si en él hay una relación binaria $\preceq$, que cumple las siguientes propiedades:

a) Reflexividad, si $x \preceq x$ para cada $x \in X$.

b) Transitividad, si $x \preceq y$ y $y \preceq z$ implican que $x \preceq z$, para $x, y, z \in X$.

c) Antisimetría, si $x \preceq y$ y $y \preceq x$ implican que $x=y$, para $x, y \in X$.

Nota 1.3. $\mathrm{A} \preceq$ se le llama relación de orden.

Ejemplo 1.4. Los siguientes son conjuntos parcialmente ordenados

1. $\mathbb{R}$ con la relación $\leq$, es decir, el orden usual en $\mathbb{R}$.

2. Dado un conjunto $X$, el conjunto potencia, denotado por $\mathscr{P}(X)$, con la relación $\subseteq$.

3. Sean $I$ un conjunto de índices y $X_{i}$ un conjunto parcialmente ordenado con relación $\preceq_{i}$, para cada $i \in I$; el producto directo $\prod_{i \in I} X_{i}$, con la relación $\preceq$, dada por $x \preceq y$, para $x, y \in \prod_{i \in I} X_{i}$, si $x_{i} \preceq_{i} y_{i}$, para cada $i \in I$.

En un conjunto parcialmente ordenado, no siempre ocurre que $x \preceq y$ o $y \preceq x$, para cualquier par $x, y$ en él, esto da lugar a lo siguiente.

Definición 1.5. Se dice que $x, y$ son comparables en $X$ si $x \preceq y$ o $y \preceq x$, en otro caso, $x$ y $y$ son incomparables.

Definición 1.6. Un conjunto parcialmente ordenado es una cadena si todos sus elementos son comparables.

Ahora, para tener un acercamiento al concepto de latiz, se dan algunas nociones de cotas en conjuntos parcialmente ordenados.

Definición 1.7. Dados un conjunto parcialmente ordenado $X$ y $X^{\prime}$ un subconjunto de este, $y \in X$ es una cota superior (inferior) de $X^{\prime}$ si $x \preceq y(y \preceq x)$, para cada $x \in X^{\prime}$, si además, se tiene que $y \in X^{\prime}$, entonces $y$ es el elemento máximo (mínimo) de $X^{\prime}$. 
Definición 1.8. Se dice que $x \in X^{\prime}$ es un elemento maximal (minimal) de $X^{\prime}$ si no existe $y \in X^{\prime}$ tal que $x \prec y(y \prec x)$.

Observación 1.9. Todo elemento máximo es maximal, de manera análoga, todo elemento mínimo es minimal.

En un conjunto parcialmente ordenado, los elementos máximos (mínimos), si existen, son únicos. En cambio, puede que en el conjunto exista más de un elemento maximal (minimal). Los elementos maximales (minimales) distintos no son comparables.

Definición 1.10. Si el conjunto de cotas superiores de $X$ tiene un elemento mínimo, esta mínima cota superior es llamada supremo de $X$, similarmente, la máxima cota inferior de $X$ es llamada ínfimo.

Notación 1.11. Considere $X$ un conjunto parcialmente ordenado, y $x, y \in X$. Si estos tienen una mínima cota superior en $X$ (join), se denota por $x \vee y$; de manera similar, la máxima cota inferior en $X$ de dichos elementos (meet), se denota $x \wedge y$.

\subsubsection{Latices, sublatices y propiedades}

En esta sección, se proporcionan los conceptos de latiz y sublatiz, estos serán usados en el capítulo 2, para caracterizar los conjuntos de perfiles de estrategias admisibles en los juegos supermodulares no cooperativos.

Usando las definiciones de la sección 1.2.1, se introducirá uno de los conceptos fundamentales de este capítulo, el de latiz. Además, se darán algunos ejemplos y observaciones respecto a estos conjuntos.

Definición 1.12. Un conjunto parcialmente ordenado que contiene la máxima cota inferior y la mínima cota superior de cualquier par de elementos se denomina latiz.

Ejemplo 1.13. Los siguientes conjuntos son latices

1. $\mathscr{P}(X)$, de un conjunto $X$, con la relación binaria $\subseteq$. Pues, para $A, B$ subconjuntos de $X, A \vee B=A \cup B$ y $A \wedge B=A \cap B$.

2. $\mathbb{R}$, con la relación de orden $\leq$, donde, $x \vee y=\operatorname{máx}\{x, y\}$ y $x \wedge y=\operatorname{mín}\{x, y\}$, para $x, y \in \mathbb{R}$.

3. $\mathbb{R}^{n}$ con la relación $\leq$, donde, para $x, y \in \mathbb{R}^{n}, x \vee y=\left(x_{1} \vee y_{1}, \ldots, x_{n} \vee y_{n}\right)$ y $x \wedge y=\left(x_{1} \wedge y_{1}, \ldots, x_{n} \wedge y_{n}\right)$. Con $x_{i} \vee y_{i}$ y $x_{i} \wedge y_{i}$ definidas en el inciso 2 .

Observación 1.14. El producto directo de latices es una latiz. 
Puede ocurrir que un subconjunto de una latiz sea tal que, para cualquier par de sus elementos el conjunto contenga su máxima cota inferior y su mínima cota superior respecto a la latiz, esto da lugar a lo siguiente.

Definición 1.15. Si $Y$ es un subconjunto de una latiz $X$ y es tal que, para cualquier par de sus elementos contiene su máxima cota inferior y su mínima cota superior, respecto de $X$, entonces $Y$ es una sublatiz de $X$.

A continuación, se darán algunos ejemplos y observaciones sobre sublatices.

Nota 1.16. El conjunto de sublatices no vacías de una latiz $X$ es denotado por $\mathscr{L}(X)$.

Ejemplo 1.17. Los siguientes son sublatices

1. Cualquier subconjunto de $\mathbb{R}$.

2. Cualquier subconjunto de una cadena es una sublatiz de esta.

Observación 1.18. Sobre sublatices.

a) Dada una latiz $X$ y $X^{\prime}$ una sublatiz de esta, si $Y$ es una sublatiz de $X^{\prime}$, entonces también lo es de $X$.

b) Si $X$ y $Y$ son latices con la misma relación de orden y tales que $X \subset Y$ no siempre ocurre que $X$ sea una sublatiz de $Y$.

Para ejemplificar la segunda parte de la observación, considere el siguiente:

Ejemplo 1.19. Sea $X$ un conjunto no vacío y $Y=\mathscr{P}(X)$, se sabe que $Y$ es una latiz con la relación binaria $\subseteq$. Considere $Z=\{\emptyset, X, A, B\}$, con $A, B \subset X, A \neq B$ y $A \neq B^{c}$.

Note que $Z \subset Y$, es una latiz con la relación $\subseteq$ pero, en $Z$ se tiene que $A \vee_{Z} B=X$ y $A \wedge_{Z} B=\emptyset$, en cambio $A \vee_{Y} B=A \cup B$ y $A \wedge_{Y} B=A \cap B$, de esto que $Z$ no sea una sublatiz de $Y$.

Los siguientes lemas son algunas propiedades básicas de latices.

Lema 1.20. Si $Y$ es una sublatiz de una latiz $X$ y $Z$ un subconjunto de $Y$, finito y no vacío, entonces $\inf _{X}\{Z\}$ y $\sup _{X}\{Z\}$ existen y son elementos de $Y$. De esto que, si $X \neq \emptyset$ es una latiz finita, entonces $X$ tiene supremo e ínfimo.

La demostración del lema 1.20 se encuentra en el apéndice A. 
Lema 1.21. Si $X$ es una latiz y $X_{\alpha}$ es una sublatiz de $X$ para cada $\alpha \in A$, entonces $\bigcap_{\alpha \in A} X_{\alpha}$ es una sublatiz de $\mathrm{X}$.

\section{DEMOSTRACIÓN}

Sean $x, y \in \bigcap_{\alpha \in A} X_{\alpha}$, se tiene que $x, y \in X_{\alpha}, \forall \alpha \in A$, y estas son sublatices de $X$, de aquí que $x \vee y, x \wedge y \in X_{\alpha}, \forall \alpha \in A$, así $x \vee y, x \wedge y \in \bigcap_{\alpha \in A} X_{\alpha}$.

De esto se concluye que $\bigcap_{\alpha \in A} X_{\alpha}$ es una sublatiz de $X$.

La siguiente definición será usada en el capítulo 2, para representar algunos conjuntos particulares de estrategias admisibles para los jugadores.

Definición 1.22. Sean $X$ y $T$ latices, $S \subset X \times T$, la sección de $S$ sobre $t$ en T, es el conjunto $S_{t}=\{x \in X:(x, t) \in S\}$, y la proyección de $S$ sobre $T$ es $\Pi_{T} S=\left\{t \in T: S_{t} \neq \emptyset\right\}$.

Ahora, se dará un resultado sobre los conjuntos dados en la definición 1.22.

Lema 1.23. Sean $X$ y $T$ latices, y $S$ una sublatiz de $X \times T$.

a) La sección $S_{t}$ de $S$ en cada $t \in T$ es una sublatiz de $X$.

b) La proyección $\Pi_{T} S$ de $S$ sobre $T$ es una sublatiz de $T$.

\section{DEMOSTRACIÓN}

a) Sea $t \in T$ y $x, y \in S_{t}$, luego $(x, t),(y, t) \in S$. Como $S$ es una sublatiz, $(x, t) \vee(y, t) \in$ $S$ y $(x, t) \wedge(y, t) \in S$, así $x \vee y, x \wedge y \in S_{t}$. De esto que $S_{t}$ sea una sublatiz de $X$.

b) Considere $t, u \in \Pi_{T} S$, se tiene que $S_{t}$ y $S_{u}$ son no vacíos; sean $s$ y $v$ en $S_{t}$ y $S_{u}$, respectivamente. $S$ es una sublatiz de $X \times T$, así que

$$
(s, t) \vee(v, u)=(s \vee v, t \vee u) \in S \quad \mathrm{y} \quad(s, t) \wedge(v, u)=(s \wedge v, t \wedge u) \in S
$$

Se sigue que, $S_{t \vee u}$ y $S_{t \wedge u}$ son no vacíos, luego $t \vee u$ y $t \wedge u$ están en $\Pi_{T} S$. Con esto se concluye que $\Pi_{T} S$ es una sublatiz de $T$. 


\section{Propiedades topológicas}

En este apartado, se define la noción de completez para latices y se da una caracterización de latices completas usando conceptos topológicos. Dichos resultados serán de utilidad para asegurar la existencia de máximos y mínimos en los conjuntos de perfiles de mejor respuesta, para juegos supermodulares cuyos perfiles de estrategias admisibles son subconjuntos de $\mathbb{R}^{n}$.

Se comenzará definiendo completez para latices, seguido de esto se darán observaciones de latices completas y algunos ejemplos.

Definición 1.24. Una latiz en la cual cada subconjunto no vacío tiene supremo e ínfimo se dice completa.

Nota 1.25. De la definición anterior y el lema 1.20, se tiene que:

a) Cualquier latiz finita es completa.

b) Una latiz completa, no vacía, tiene supremo e ínfimo.

Ejemplo 1.26. Las siguientes son latices completas.

1. Los intervalos cerrados en $\mathbb{R}$.

2. $\mathscr{P}(X)$, para $X \neq \emptyset$.

Haciendo uso del concepto de sublatiz y del de completez, surge la siguiente definición.

Definición 1.27. Si $Y$ es una sublatiz de una latiz $X$ y es tal que, para cada $Z \subset Y$, $Z \neq \emptyset, \sup _{X} Z, \inf _{X} Z \in Y$, entonces $Y$ es una sublatiz subcompleta de $X$.

Ejemplo 1.28. Tomando las latices completas del ejemplo 1.26, se presentan las siguientes sublatices subcompletas de estas.

1. Para los intervalos cerrados en $\mathbb{R}$, los conjuntos de la forma $[a, b] \cap \mathbb{N}$.

2. Para $\mathscr{P}(X)$, la colección $\left\{A, A^{c}, \emptyset, X\right\}$, con $A \subset X$.

Observación 1.29. Sobre latices completas.

a) Si $X$ es una latiz y $Y$ una sublatiz subcompleta de esta, entonces $\sup _{X} Z=$ $\sup _{Y} Z \operatorname{e~inf}_{X} Z=\inf _{Y} Z$, para cualquier $Z \subset Y, Z \neq \emptyset$. Además, $Y$ es una latiz completa. 
b) Si $X$ y $Y$ son latices completas con la misma relación de orden y $Y \subset X$, entonces $Y$ no necesariamente es una sublatiz subcompleta de $X$.

c) La intersección de una latiz completa (sublatiz subcompleta) y un intervalo cerrado, es una latiz completa (sublatiz subcompleta).

Para mostrar b) de la observación anterior, se tiene el siguiente.

Ejemplo 1.30. Recordando el ejemplo 1.19, para $X \neq \emptyset, \mathscr{P}(X)$ es una latiz completa. Si $Z=\{A, B, X, \emptyset\}$, con $A \neq B, A \neq B^{c}$ y $A, B \subset X$. Note que $Z \subset \mathscr{P}(X)$ y $Z$ es una latiz completa.

Considere $Y=\{A, B\}, Y \subset Z$, observe que $\sup _{Z} Y=X \neq \sup _{\mathscr{P}(X)} Y=A \cup B$. De esto que $Z$ no sea una sublatiz subcompleta de $\mathscr{P}(X)$.

El siguiente teorema, cuya demostración se puede revisar en el apéndice A, muestra que subcompletez es equivalente a compacidad en la topología usual de $\mathbb{R}^{n}$. Este resultado y sus implicaciones, serán de utilidad en el capítulo 2.

Teorema 1.31. Una sublatiz de $\mathbb{R}^{n}$ es subcompleta si y solo si es compacta.

Del teorema recien eunciado, se puede dedudir el siguiente resultado.

Corolario 1.32. Una sublatiz de $\mathbb{R}^{n}$, compacta y no vacía, tiene un elemento máximo y un elemento mínimo.

El lema que se enuncia a continuación, provee un resultado para las sublatices no vacías y cerradas que son acotadas, ya sea superior o inferiormente.

Lema 1.33. Una sublatiz cerrada de $\mathbb{R}^{n}$ que es acotada superiormente (inferiormente), tiene un elemento máximo (mínimo).

La demostración del lema 1.33, se puede encontrar en [10] (página 31, corolario 2.3.1).

\subsubsection{Orden inducido de un conjunto}

En esta sección, se darán propiedades y caracterizaciones de una relación de orden inducido en sublatices no vacías de una latiz. Estos resultados serán de utilidad para definir algunas propiedades de las correspondencias de mejor respuesta en los juegos supermodulares no cooperativos.

Se comenzará definiendo el orden inducido de un conjunto y se proveerán algunos atributos de este. 
Definición 1.34. Sea $X$ una latiz con relación de orden $\preceq$. El orden inducido del conjunto $\sqsubseteq$, se define sobre la colección de elementos no vacíos del conjunto potencia de $X$, y es tal que para $A, B \in \mathscr{P}(X) \backslash\{\emptyset\}, A \sqsubseteq B$, si $x \in A$ y $y \in B$ implican que, $x \wedge y \in A$ y $x \vee y \in B$.

Los singuletes en $X$ son ordenados por $\sqsubseteq$ si y sólo si, los elementos son ordenados por $\preceq$; es decir, para $a, b \in X,\{a\} \sqsubseteq\{b\}$ si y sólo si $a \preceq b$.

Lema 1.35. Sean $X$ una latiz y $Y, Z$ subconjuntos no vacíos de $X$, tales que $Y \sqsubseteq Z$, si $\sup _{X}(Y)$ y $\sup _{X}(Z)\left(\inf _{X}(Y)\right.$ e inf $\left.\operatorname{in}_{X}(Z)\right)$ existen, entonces, se tiene que $\sup _{X}(Y) \preceq \sup _{X}(Z)\left(\inf _{X}(Y) \preceq \inf _{X}(Z)\right)$.

DEMOSTRACIÓN

Considere $y \in Y$ y $z \in Z$. Como $Y \sqsubseteq Z$, se tiene que $y \wedge z \in Y$ y $y \vee z \in Z$, luego $y \preceq y \vee z \preceq \sup _{X}\{Z\}$, esto sucede para todo $y \in Y$. $\operatorname{Así}_{\sup _{X}}\{Y\} \preceq \sup _{X}\{Z\}$.

De manera análoga, se prueba que inf $\operatorname{in}_{X}\{Y\} \preceq y \wedge z \preceq z, \forall z \in Z$, entonces ínf ${ }_{X}\{Y\} \preceq$ $\inf _{X}\{Z\}$.

A continuación, se define lo que es una correspondencia (o multifunción), este concepto es importante ya que, posteriormente se dará una caracterización de los puntos de equilibrio en juegos no cooperativos basada en una correspondencia particular.

Definición 1.36. Una función cuyo rango está contenido en $\mathscr{P}(X)$, para algún conjunto $X$, es llamada correspondencia.

Nota 1.37. Una correspondencia $\mathrm{S}_{t}: T \rightarrow \mathscr{P}(X)$ se dice creciente (decreciente) en $t$, para $t \in T$, si:

a) El dominio $T$, es un conjunto parcialmente ordenado.

b) El rango, $\left\{S_{t}: t \in T\right\} \subset \mathscr{L}(X)$, con $X$ una latiz y $\mathscr{L}(X)$ parcialmente ordenado con relación $\sqsubseteq$.

c) Los conjuntos $S_{t}$ son crecientes (decrecientes), i.e., $t \preceq r \Rightarrow S_{t} \sqsubseteq S_{r}\left(S_{r} \sqsubseteq S_{t}\right)$.

Teorema 1.38. Sean $X$ y $T$ latices.

a) Si $S$ es una sublatiz de $X \times T$ entonces, la sección $S_{t}$ de $S$ en $t, t \in T$, es creciente en $t$ sobre la proyección $\Pi_{T} S$ de $S$ en $T$. 
b) Si $T$ es una cadena, $S_{t} \subset X, \forall t \in T, \mathrm{~S}_{t}$ es creciente en los $t$ con $S_{t} \neq \emptyset$ y $S=\left\{(x, t): t \in T, x \in S_{t}\right\}$, entonces $S$ es una sublatiz de $X \times T$.

\section{DEMOSTRACIÓN}

a) Se probará que para $t, r \in \Pi_{T} S$, con $t \preceq r$, se tiene que $S_{t} \sqsubseteq S_{r}$.

Considere $t, r \in \Pi_{T} S$, tales que $t \preceq r$, y sean $a \in S_{t}, b \in S_{r}$, se tiene que

$$
(a, t) \wedge(b, r)=(a \wedge b, t) \quad \mathrm{y} \quad(a, t) \vee(b, r)=(a \vee b, r),
$$

de esto que $a \wedge b \in S_{t}$ y $a \vee b \in S_{r}$, así los conjuntos $S_{t}$ con crecientes en $t \in \Pi_{T} S$.

b) Se probará que $a \vee b, a \wedge b \in S$, para cualesquiera $a, b \in S$.

Sean $(a, x)$ y $(b, y)$ en $S$, sin pérdida de generalidad, se puede suponer que $x \preceq y$, pues $T$ es una cadena, además $S_{x} \sqsubseteq S_{y}$, con $a \in S_{x}$ y $b \in S_{y}$. Luego, $a \wedge b \in S_{x}$ y $a \vee b \in S_{y}$, de aquí que $a \wedge b$ y $a \vee b$ estén en $S$ y se concluye que $S$ es una sublatiz.

La siguiente observación se usa en la prueba de algunos resultados de la sección 1.2.4.

Observación 1.39. Sean $X, Y$ conjuntos parcialmente ordenados, no vacíos. Considere $f: X \rightarrow \mathscr{P}(Y)$, si $f$ es una correspondencia creciente y $y \preceq x$ en $X$ entonces, para cualquier $s \in f(x)$ existe $t \in f(y)$ tal que, $t \preceq s$.

\subsubsection{Puntos fijos}

Se definirán puntos fijos para funciones y correspondencias, además se darán algunas propiedades de estos cuando las correspondencias tienen ciertas características. Este apartado es importante pues, los puntos de equilibrio en juegos no cooperativos se pueden ver como puntos fijos de la correspondencia de mejor respuesta.

Se inicia la sección con el concepto de punto fijo para una función y para una correspondencia.

Definición 1.40. Sea $f$ una función de un conjunto $X$ en sí mismo, si $x^{*} \in X$ satisface que $f\left(x^{*}\right)=x^{*}$ entonces, $x^{*}$ es un punto fijo de $f$. Similarmente, si $f: X \rightarrow \mathscr{P}(X)$ es una correspondencia tal que, para $x^{*} \in X$, $x^{*} \in f\left(x^{*}\right)$, decimos que $x^{*}$ es un punto fijo de $f$. 
Teniendo la definición de punto fijo en correspondencias, el siguiente resultado se tiene cuando el conjunto $X$ es una latiz completa, no vacía, y la correspondencia cumple algunas hipótesis sobre su rango e imagen.

Teorema 1.41. Sea $X$ una latiz completa y no vacía, Y $X \rightarrow \mathscr{L}(X)$ una correspondencia creciente, donde $\mathscr{L}(X)$ tiene el orden inducido $\sqsubseteq$ y $Y(x)$ es una sublatiz subcompleta para cada $x \in X$. Entonces:

a) El conjunto de puntos fijos de $Y$ en $X$ es no vacío, además, tiene elementos máximo y mínimo, dados por $\sup _{X}\{x \in X: Y(x) \cap[x, \infty) \neq \emptyset\}$ e $\inf _{X}\{x \in$ $X: Y(x) \cap(-\infty, x] \neq \emptyset\}$, respectivamente.

b) El conjunto de puntos fijos de $\mathrm{Y}$ en $X$ es una latiz completa y no vacía.

La demostración del teorema 1.41 se puede ver en el apéndice A.

Ahora, para correspondencias crecientes cuyo dominio es un producto de conjuntos con ciertas propiedades, se tiene lo siguiente:

Teorema 1.42. Sean $X$ una latiz completa, $T$ un conjunto parcialmente ordenado y $\mathrm{Y}: X \times T \rightarrow \mathscr{L}(X)$ una correspondencia creciente con $Y(x, t)$ una sublatiz subcompleta para cada $(x, t) \in X \times T$.

a) Para cada $t \in T$, la correspondencia $Y$ tiene un máximo (mínimo) punto fijo.

b) El máximo (mínimo) punto fijo de $\mathrm{Y}$ es creciente en $T$.

c) Si también se supone que $\sup \{Y(x, t)\}<\inf \{Y(x, s)\}, \forall x \in X$ y $t \prec s$, entonces el máximo (mínimo) punto fijo de $Y$ es estrictamente creciente en $T$.

Cuando las imágenes de la correspondencia Y son singuletes, los resultados anteriores se pueden reescribir como:

Corolario 1.43. Sea $f: X \rightarrow X$, una función creciente de una latiz completa y no vacía, en sí misma.

a) El conjunto de puntos fijos de $f$ es no vacío y sus elementos máximo y mínimo se pueden describir $\operatorname{como} \sup \{x \in X: x \preceq f(x)\}$ e $\inf \{x \in X: f(x) \preceq x\}$, respectivamente.

b) El conjunto de puntos fijos de $f$ es una latiz completa y no vacía. 
Corolario 1.44. Sean $X$ una latiz completa y no vacía, $T$ un conjunto parcialmente ordenado y $f: X \times T \rightarrow X$ una función creciente.

a) La función $f$ tiene un máximo (mínimo) punto fijo, para cada $t \in T$.

b) El máximo (mínimo) punto fijo de $f$ es creciente en $T$.

c) Si además, $f$ es estrictamente creciente en $T$, para cada $x \in X$, entonces el máximo (mínimo) punto fijo de $f$ es estrictamente creciente en $T$.

\subsection{Funciones supermodulares}

En este apartado se definirán las funciones supermodulares, dichas funciones son de interés ya que, la supermodularidad es característica de las utilidades en los juegos supermodulares no cooperativos.

Inicialmente se dará el concepto de diferencias crecientes y algunas observaciones sobre este. Las funciones con diferencias crecientes son mencionadas pues, en ciertos casos, la supermodularidad se puede deducir de esta propiedad.

Definición 1.45. Sean $X$ y $T$ conjuntos parcialmente ordenados y una función $f: S \rightarrow \mathbb{R}$, con $S \subset X \times T$. Si $f(x, t)-f(x, r)$ es creciente (decreciente, estrictamente creciente o estrictamente decreciente) en $x \in S_{t} \cap S_{r}$, para cualesquiera $t, r \in T$, con $r \preceq t$, decimos que $f$ tiene diferencias crecientes (decrecientes, estrictamente crecientes o estrictamente decrecientes).

Nota 1.46. La definición de diferencias crecientes es válida para ambas entradas de $f$ pues,

$$
f(x, t)-f(x, r) \leq f(y, t)-f(y, r) \Leftrightarrow f(y, r)-f(x, r) \leq f(y, t)-f(x, t) .
$$

Cuando el dominio de $f$ es subconjunto de un producto arbitrario de conjuntos parcialmente ordenados, la condición de diferencias crecientes se decribe de la siguiente manera.

Observación 1.47. La función $f$ tiene diferencias crecientes cuando el dominio de $f$ es subconjunto de un producto arbitrario de conjuntos parcialmente ordenados, es decir, $f: X \rightarrow \mathbb{R}$, con $X \subset \prod_{\alpha \in A} X_{\alpha}$ y para cualesquiera $\alpha, \beta \in A, \alpha \neq \beta$, y cualquier $x_{\gamma} \in X_{\gamma}$, con $\gamma \in A \backslash\{\alpha, \beta\}$, $f$ tiene diferencias crecientes en los $\left(x_{\alpha}, x_{\beta}\right)$ pertenecientes a la sección de $X$ en $x_{\gamma}$. 
A continuación, se da una equivalencia de diferencias crecientes cuando la función es derivable, este resultado será de utilidad en el capítulo 3.

Teorema 1.48. Dada la función $f: \mathbb{R}^{n} \rightarrow \mathbb{R}$, diferenciable en $\mathbb{R}^{n}$, esta tiene diferencias crecientes si y sólo si $\frac{\partial f(x)}{\partial x_{i}}$ es creciente en $x_{j}$, para cualquier $x \in \mathbb{R}^{n}$, con $j \neq i$. Equivalentemente, si $f$ tiene segunda derivada, se dice que tiene diferencias crecientes si y sólo si $\frac{\partial^{2} f(x)}{\partial x_{i} \partial x_{j}} \geq 0$, con $i \neq j$, para cualquier $x \in \mathbb{R}^{n}$.

Demostración

Por simplicidad, la prueba se hará para $n=2$, el caso general es análogo.

Se dice que la función $f$ tiene diferencias crecientes si, $f(\cdot, b)-f(\cdot, a)$ es una función creciente, con $b>a, a, b \in \mathbb{R}$; en otra palabras, si

$$
\frac{\partial}{\partial y}[f(y, b)-f(y, a)] \geq 0 \text { es decir, si } \frac{\partial f(y, b)}{\partial y}>\frac{\partial f(y, a)}{\partial y}
$$

Dicho de otro modo, si $\frac{\partial f(y, a)}{\partial y}$ es una función creciente en $a$, lo cual equivale a decir que $\frac{\partial^{2} f(y, a)}{\partial y \partial a} \geq 0$

Definición 1.49. Sea $X$ una latiz. Considere $f: X \rightarrow \mathbb{R}$, decimos que $f$ es supermodular en $X$ si, para cualesquiera $x, y \in X, f$ es tal que

$$
f(x)+f(y) \leq f(x \vee y)+f(x \wedge y)
$$

Si la desigualdad es estricta, se dice que $f$ es estrictamente supermodular. Similarmente, decimos que $f$ es submodular en $X$ si $-f$ es supermodular.

Nota 1.50. Una función que es supermodular y submodular es llamada valuación.

El siguiente resultado, cuya prueba se puede revisar en [10] (página 44, teorema 2.6.1), caracteriza las funciones con diferencias crecientes en sublatices.

Teorema 1.51. Sean $A$ un conjunto de índices, $X_{\alpha}$ una latiz, para cada $\alpha \in A$, y $X \subset \prod_{\alpha \in A} X_{\alpha}$ una sublatiz. Si $f$ es supermodular en $X$, entonces $f$ tiene diferencias crecientes en $X$. 
A continuación, se darán dos resultados que identifican la supermodularidad en términos de diferencias crecientes cuando el dominio de la función tiene ciertas características; seguido de esto, se muestran algunos ejemplos de funciones supermodulares.

Teorema 1.52. Sea $X_{i}$ una latiz, para $i=1, \ldots, n$. Si $f: \prod_{i=1}^{n} X_{i} \rightarrow \mathbb{R}$ es una función con diferencias crecientes y supermodular en cada entrada, entonces $f$ es supermodular en $\prod_{i=1}^{n} X_{i}$.

La demostración del teorema 1.52 se encuentra en [10] (página 45, teorema 2.6.2).

Corolario 1.53. Considere $N=\{1, \ldots, n\}$, con $n \in \mathbb{N}, X_{i}$ una cadena para cada $i \in$ $N$ y $f$ una función con diferencias crecientes en $\prod_{i=1}^{n} X_{i}$, entonces $f$ es supermodular en $\prod_{i=1}^{n} X_{i}$.

Observación 1.54. El resultado anterior no es cierto cuando $N$ no es finito.

Ejemplo 1.55. Las siguientes funciones son supermodulares.

1. Sea $X$ una cadena, cualquier función $f: X \rightarrow \mathbb{R}$ es una valuación.

2. La función $f\left(x_{1}, x_{2}\right)=x_{1} x_{2}$ es supermodular en $\mathbb{R}^{2}$.

3. La función $f\left(x_{1}, \ldots, x_{n}\right)=\operatorname{mín}\left\{\alpha_{i} x_{i}: i=1, \ldots, n\right\}$, con $\alpha_{i} \geq 0$, para $i=$ $1, \ldots, n$, es supermodular en $\mathbb{R}^{n}$.

Ahora, se presenta el concepto de log-supermodularidad, este será útil para identificar la supermodularidad en funciones positivas.

Definición 1.56. Sea $X$ una latiz y $f: X \rightarrow \mathbb{R}^{+}$. Se dice que $f$ es log-supermodular si y solo si

$$
f(x) f(y) \leq f(x \vee y) f(x \wedge y)
$$

para cualesquiera $x, y \in X$.

Contando con la definición de función log-supermodular, el siguiente resultado relaciona log-supermodularidad y supermodularidad en funciones crecientes.

Teorema 1.57. Considere $X$ una latiz y $f: X \rightarrow \mathbb{R}^{+}$. Si $f$ es creciente y logsupermodular, entonces $f$ es supermodular.

DEMOSTRACIÓN

Sean $x, y \in X$, como $f$ es log-supermodular, se tiene que

$$
f(x) f(y) \leq f(x \wedge y) f(x \vee y)
$$


así

$$
\frac{f(x)}{f(x \wedge y)}-1 \leq \frac{f(x \vee y)}{f(y)}-1
$$

Ahora, como $f$ es creciente, se tiene que $f(y) \geq f(x \wedge y)$, pues $y \geq x \wedge y$. Así

$$
\frac{f(x)}{f(x \wedge y)}-1 \leq\left(\frac{f(x \vee y)}{f(y)}-1\right) \frac{f(y)}{f(x \wedge y)},
$$

de esto que

$$
f(x)-f(x \wedge y) \leq f(x \vee y)-f(y) .
$$

$\mathrm{Y}$ se concluye que $f$ es supermodular.

\subsubsection{Conjunto de soluciones óptimas}

A continuación, se define el conjunto de soluciones óptimas de una función, este concepto será usado al definir la correspondencia de mejor respuesta en juegos supermodulares no cooperativos.

Definición 1.58. Dado el problema de maximizar una función real valuada, $f$ : $X \rightarrow \mathbb{R}$, el conjunto de soluciones óptimas de $f$ se define como

$$
\operatorname{argmáx}_{x \in X} f(x)=\{x \in X: f(y) \leq f(x), \forall y \in X\} .
$$

Los resultados que se enunciarán a continuación, serán de utilidad para asegurar la existencia de equilibrios en juegos supermodulares no cooperativos.

Teorema 1.59. Sean $X$ una latiz y $f: X \rightarrow \mathbb{R}$ una función supermodular, entonces $\operatorname{argmáx}_{x \in X} f(x)$ es una sublatiz de $X$.

Demostración

Sean $x, y \in \operatorname{argmáx}_{x \in X} f(x)$, como $f$ es supermodular, se tiene que

$$
f(x)+f(y) \leq f(x \wedge y)+f(x \vee y),
$$

así,

$$
0 \leq f(x)-f(x \wedge y) \leq f(x \vee y)-f(y) \leq 0,
$$

donde la primera y la última desigualdad se dan porque $x$ y $y$ maximizan la función $f$, se sigue que $x \vee y$ y $x \wedge y$ están en $\operatorname{argmáx}_{x \in X} f(x)$. 
Definición 1.60. Sea $X$ un espacio métrico y $f: X \rightarrow \mathbb{R}$, se dice que $f$ es semicontinua superiormente en $x_{0} \in X$ si, para $c \in \mathbb{R}$ con $c<f\left(x_{0}\right)$, existe $\delta>0$ tal que, si $d\left(x, x_{0}\right)<\delta$ entonces $c<f(x)$.

Corolario 1.61. Sea $X \neq \emptyset$ una latiz y $f: X \rightarrow \mathbb{R}$ una función supermodular.

a) Si $X$ es finita, entonces $\operatorname{argmáx}_{x \in X} f(x)$ es una sublatiz subcompleta, no vacía, de $X$.

b) Si $X$ es una sublatiz compacta de $\mathbb{R}^{n}$ y $f$ es semicontinua superiormente en $X$, entonces $\operatorname{argmáx}_{x \in X} f(x)$ es una sublatiz de $\mathbb{R}^{n}$, subcompleta, compacta y no vacía.

Teorema 1.62. Sean $X$ y $Y$ latices, $S \subset X \times Y$ una sublatiz y $f: S \rightarrow \mathbb{R}$ una función supermodular. Si $g(y)=\sup _{x \in S_{y}} f(x, y)$ es finito en $\Pi_{Y} S$, entonces $g$ es supermodular.

Demostración

Considere $y, z \in \Pi_{Y} S$ y sean $a \in S_{y}$ y $b \in S_{z}$. La función $f$ es supermodular, de esto que

$$
f(a, y)+f(b, z) \leq f(a \vee b, y \vee z)+f(a \wedge b, y \wedge z)
$$

tomando supremos, se tiene que

$$
g(y)+g(z) \leq g(y \wedge z)+g(y \vee z)
$$

y se concluye lo deseado.

Los siguientes resultados dan condiciones suficientes para que el conjunto de soluciones óptimas, $\operatorname{argmáx}_{x \in S_{t}} f(x, t)$, sea creciente.

Lema 1.63. Sean $X$ una latiz, $T$ un conjunto parcialmente ordenado y $S_{t} \subset X$, para cada $t \in T$. Si los conjuntos $S_{t}$ son crecientes en $t \mathrm{y}$,

$$
f(x, t)+f(y, r) \leq f(x \wedge y, t)+f(x \vee y, r),
$$

para cualesquiera $t, r \in T$, con $t \preceq r$ y $x \in S_{t}, y \in S_{r}$, entonces $\operatorname{argmáx}_{x \in S_{t}} f(x, t)$ es creciente en $t \in\left\{t \in T: \operatorname{argmáx}_{x \in S_{t}} f(x, t) \neq \emptyset\right\}$.

Demostración 
Se probará que, para $x \in \operatorname{argmáx}_{a \in S_{t}} f(a, t)$ y $y \in \operatorname{argmáx}_{a \in S_{r}} f(a, r)$, con $t \preceq r$, en $\left\{t \in T: \operatorname{argmáx}_{x \in S_{t}} f(x, t) \neq \emptyset\right\}$, se sumple que $x \wedge y \in \operatorname{argmáx}_{a \in S_{t}} f(a, t)$ y $x \vee y \in \operatorname{argmáx}_{a \in S_{r}} f(a, r)$.

El conjunto de soluciones óptimas de $f$ es una sublatiz, para cada $t \in T$. Considere $t, r \in\left\{t \in T: \operatorname{argmáx}_{x \in S_{t}} f(x, t) \neq \emptyset\right\}$, tales que $t \preceq r$.

Ahora, tome $x \in \operatorname{argmáx~}_{a \in S_{t}} f(a, t)$ y $y \in \operatorname{argmáx~}_{a \in S_{r}} f(a, r)$. Como $S_{t} \sqsubseteq S_{r}$, se tiene que $x \wedge y \in S_{t}$ y $x \vee y \in S_{r}$. Luego, de (1.2) se sigue que

$$
0 \leq f(x, t)-f(x \wedge y, t) \leq f(x \vee y, r)-f(y, r) \leq 0 .
$$

De esto que $f(x, t)=f(x \wedge y, t)$ y $f(x \vee y, r)=f(y, r)$. Así $x \wedge y \in \operatorname{argmáx}_{a \in S_{t}} f(a, t)$ y $x \vee y \in \operatorname{argmáx}_{a \in S_{r}} f(a, r)$.

Teorema 1.64. Sean $X$ una latiz, $T$ un conjunto parcialmente ordenado y para cada $t \in T, S_{t} \subset X$, subconjunto creciente en $t$. Considere $f(x, t)$ una función

supermodular en $X$, con diferencias crecientes en $X \times T$. Entonces $\operatorname{argmáx}_{x \in S_{t}} f(x, t)$ es creciente en $t \in\left\{t \in T: \operatorname{argmáx}_{x \in S_{t}} f(x, t) \neq \emptyset\right\}$.

Teorema 1.65. Sean $X$ y $T$ latices y $S \subset X \times T$, una sublatiz. Tome $S_{t}$ la sección de $S$ en $t \in T$ y $f$ una función supermodular en $S$, entonces $\operatorname{argmáx}_{x \in S_{t}} f(x, t)$ es creciente para $t \in\left\{t \in T: \operatorname{argmáx}_{x \in S_{t}} f(x, t) \neq \emptyset\right\}$.

Teorema 1.66. Si se cumplen las hipótesis del teorema 1.64 o del teorema 1.65 y cada $S_{t} \neq \emptyset$, con $S_{t}$ finito o subconjunto compacto de $\mathbb{R}^{m}$ y $f$ es semicontinua superiormente, para cada $t \in T$, entonces $\operatorname{argmáx}_{x \in S_{t}} f(x, t)$ es una sublatiz no vacía de $X$ y sus elementos máximo y mínimo son crecientes en $t \in T$.

Las pruebas de los teoremas 1.64, 1.65, 1.66, se encuentran en [10] (páginas 77-78, teoremas 2.8.1, 2.8.2, 2.8.3).

\subsection{Conclusiones}

En este capítulo se revisó la teoría necesaria para poder caracterizar los conjuntos de estrategias admisibles y las funciones de utilidad en los juegos supermodulares no cooperativos, también se enunciaron algunos resultados que serán de utilidad para asegurar la existencia de puntos fijos en correspondencias que se definirán posteriormente, los cuales, como se mostrará después, determinarán equilibrios en los juegos de nuestro interés. 


\section{Capítulo 2}

\section{Juegos Supermodulares no Cooperativos}

\subsection{Introducción}

En este capítulo, se describirá lo que son los juegos supermodulares no cooperativos, se hace énfasis en este tipo particular de juegos ya que, en ellos, es posible aproximar equilibrios mediante algoritmos si se agregan algunos supuestos a las funciones de utilidad y a los conjuntos de estrategias admisibles.

Inicialmente se definirá lo que son los juegos finitos no cooperativos y algunos conceptos importantes, como son, punto de equilibrio, correspondencia de mejor respuesta, entre otros. Luego, se presentará el teorema de Nash que asegura la existencia de equilibrios en los juegos finitos.

Seguido de esto, se definirán los juegos supermodulares no cooperativos y se darán resultados que aseguran la existencia de puntos de equilibrio, en este tipo particular de juegos. Finalmente, se enunciarán dos algoritmos para generar sucesiones de perfiles de estrategias admisibles y resultados que afirman que las sucesiones de estrategias admisibles generadas convergen a puntos de equilibrio en dichos juegos.

La teoría de este capítulo fue revisada en [4], [6], [7] y [10]. 


\subsection{Definición de juego supermodular}

Para definir los juegos supermodulares, se necesita el concepto de juego no cooperativo y antes de definir este, vale la pena mencionar que, en un juego, cada uno de los jugadores tiene un conjunto de posibles decisiones, dicho conjunto es conocido como el conjunto de estrategias admisibles del jugador.

Nota 2.1. Este trabajo se enfoca en los juegos con un número finito de jugadores entonces, el conjunto de jugadores se considera de la forma $N=\{1, \ldots, n\}, n \in \mathbb{N}$.

Ahora, considerando los conjuntos de estrategias admisibles de cada jugador, podemos definir lo siguiente.

Definición 2.2. Para cada $i \in N$, sea $S_{i}$ el conjunto de estrategias admisibles del jugador $i$, con $\left|S_{i}\right|=m_{i} \in \mathbb{N}$. $S \subset \prod_{i=1}^{n} S_{i}$, es llamado conjunto de perfiles de estrategias admisibles, los perfiles de estrategias (estrategias conjuntas) son $\mathbf{x}=$ $\left(x_{1}, \ldots, x_{n}\right)$, donde $x_{i}$, la estrategia del jugador $i$, es un vector de tamaño $m_{i}, \forall i \in N$.

Ahora, se puede definir lo que es un juego no cooperativo de la siguiente manera.

Definición 2.3. Un juego no cooperativo es una triada $\left(N, S,\left\{R_{i}: i \in N\right\}\right)$, donde $N \neq \emptyset$ es el conjunto de jugadores, $S$ es el conjunto de perfiles estrategias admisibles y $\left\{R_{i}: i \in N\right\}$ es la colección de funciones de pago correspondientes a cada jugador, con $R_{i}: S \rightarrow \mathbb{R}$.

A continuación, se introduce algo de notación para facilitar el enunciado de resultados posteriores.

Nota 2.4. Dado un perfil de estrategias $\mathbf{x} \in S$ y $y_{i} \in S_{i}$.

a) $x_{-i}$, es el vector de estrategias correspondientes a los jugadores en el conjunto $N \backslash\{i\}$.

b) $\left(\mathbf{x} ; y_{i}\right)$, es el perfil de estrategias donde la estrategia del $i$-ésimo jugador se cambia a $y_{i}$ y los demás jugadores mantienen su estrategia en $\mathbf{x}$.

c) $S_{i}\left(x_{-i}\right)=\left\{y_{i}:\left(x_{-i} ; y_{i}\right) \in S\right\}$, denota el conjunto de estrategias admisibles para el jugador $i$, dadas las estrategias $x_{-i}$ de los demás jugadores, i.e. $S_{i}\left(x_{-i}\right)$ es la sección de $S$ en $x_{-i}$.

d) $S_{-i}=\left\{x_{-i}: S_{i}\left(x_{-i}\right) \neq \emptyset\right\}$, la colección de vectores $x_{-i}$ tales que, existe $y_{i} \in S_{i}$ con $\left(x_{-i} ; y_{i}\right) \in S$. En otras palabras, $S_{-i}$ es la proyección de $S$ en las estrategias de todos los jugadores distintos de $i$. 
e) $S(\mathbf{x})=\left(\prod_{i=1}^{n} S_{i}\left(x_{-i}\right)\right) \cap S$.

Observación 2.5. $S=\prod_{i=1}^{n} S_{i}$ si y sólo si $S(\mathbf{x})=S$, para todo perfil de estrategias admisibles $\mathbf{x} \in S$.

Otro concepto de suma importancia en la teoría juegos es el de punto de equilibrio, que ahora se presenta:

Definición 2.6. Un perfil de estrategias admisibles $\mathrm{x}$ es llamado punto de equilibrio si

$$
R_{i}\left(x_{-i} ; y_{i}\right) \leq R_{i}(\mathbf{x}), \quad \forall y_{i} \in S_{i}\left(x_{-i}\right),
$$

en otras palabras, dado un punto de equilibrio, ningún jugador puede incrementar su pago cambiando de estrategia si las estrategias de los demás jugadores se mantienen fijas.

Con las definiciones y notación previa, se procede a definir los juegos supermodulares no cooperativos.

Definición 2.7. Un juego no cooperativo $\left(N, S,\left\{R_{i}: i \in N\right\}\right)$ se dice supermodular si, $S \subset \mathbb{R}^{m}$ es una sublatiz, la función de pago $R_{i}\left(x_{-i} ; y_{i}\right)$ es supermodular en $y_{i} \in S_{i}$, para cada $x_{-i} \in S_{-i}, \forall i \in N$; $R_{i}\left(x_{-i} ; y_{i}\right)$ tiene diferencias crecientes en $\left(x_{-i} ; y_{i}\right)$.

Las definiciones 2.8 y 2.10, nos serán de utilidad para caracterizar los puntos de equilibrio en los juegos supermodulares no cooperativos.

Definición 2.8. Sea $x_{-i} \in S_{-i}$, la correspondencia de mejor respuesta para el jugador $i$, es el conjunto de estrategias óptimas para el jugador $i$, cuando las estrategias de los demás jugadores están dadas por $x_{-i}$. Dicha correspondencia se puede describir como

$$
Y_{i}\left(x_{-i}\right)=\operatorname{argmáx}_{y_{i} \in S_{i}\left(x_{-i}\right)} R_{i}\left(x_{-i} ; y_{i}\right) .
$$

Nota 2.9. El conjunto de estrategias óptimas para el jugador $i$, cuando las estrategias de los demás jugadores están dadas por $x_{-i}$, se puede ver como el conjunto de soluciones óptimas de $R_{i}\left(x_{-i} ; \cdot\right)$.

Definición 2.10. Sean $\mathbf{x} \in S$ y $\mathbf{y} \in S(\mathbf{x})$, considere $g(\mathbf{y}, \mathbf{x})=\sum_{i \in N} R_{i}\left(x_{-i} ; y_{i}\right)$. Para cada perfil de estrategias admisibles, $\mathbf{x} \in S$, la correspondencia de mejor respuesta conjunta está dada por

$$
Y(\mathbf{x})=\operatorname{argmáx}_{\mathbf{y} \in S(\mathbf{x})} g(\mathbf{y}, \mathbf{x}),
$$

este es el conjunto de todos los perfiles de estrategias admisibles tales que, la estrategia del jugador $i$ es admisible dada $x_{-i}$ y la suma de los pagos de todos los jugadores es maximizada, pues deciden cambiar a la estrategia correspondiente en $\mathbf{y}$, en lugar de mantener la estrategia $x_{i}$, y esto sucede $\forall i \in N$. 


\subsection{Existencia de puntos de equilibrio}

En este apartado, se presentarán resultados que aseguran la existencia de puntos de equilibrio en juegos supermodulares no cooperativos.

Además, se dará la caracterización de estos como puntos fijos de la correspondencia de mejor respuesta conjunta y se mostrarán algunos resultados de las correspondencias de mejor respuesta cuando las funciones de pago y el conjunto de perfiles de estrategias cumplen ciertas propiedades.

Primeramente, se darán algunas propiedades de la correspondencia de mejor respuesta para cada jugador y la correspondencia de mejor respuesta conjunta cuando se agregan algunas condiciones al conjunto de perfiles de estrategias admisibles y a las funciones de pago.

Lema 2.11. Considere un juego supermodular $\left(N, S,\left\{R_{i}: i \in N\right\}\right)$ en el cual, el conjunto de estrategias admisibles $S$, es compacto y no vacío, la función de pago $R_{i}\left(x_{-i} ; y_{i}\right)$ es semicontinua superiormente en $y_{i} \in S_{i}\left(x_{-i}\right), \forall x_{-i} \in S_{-i}, i \in N$. Entonces

a) Para cada jugador $i$, la correspondencia de mejor respuesta $Y_{i}\left(x_{-i}\right)$ es creciente en $x_{-i} \in S_{-i}$; además, tiene elementos máximo y mínimo y estos son funciones crecientes de $S_{-i}$ en $S_{i}$. El conjunto $Y_{i}\left(x_{-i}\right)$ de mejores respuestas para cada jugador $i$ es una sublatiz subcompleta, compacta y no vacía de $\mathbb{R}^{m_{i}}$, para todo $x_{-i} \in S_{-i}$.

b) La correspondencia de mejor respuesta conjunta $Y(\mathbf{x})$ es creciente para $\mathbf{x} \in$ $S$. Los elementos máximo y mínimo de dicha correspondencia son funciones crecientes de $S$ en $S$. El conjunto $Y(\mathbf{x})$ de perfiles de mejor respuesta, es una sublatiz subcompleta, compacta y no vacía de $\mathbb{R}^{m}$, para cada $\mathbf{x} \in S$.

Un bosquejo de la demostración del resultado anterior se puede ver en el apéndice A.

Ahora, se presentará un resultado de J. Nash [6], que nos asegura la existencia de equilibrios en juegos con conjunto de jugadores y conjunto de perfiles de estrategias finitos.

Teorema 2.12. Todo juego con número finito de jugadores y conjunto de perfiles de estrategias finito tiene un punto de equilibrio. 
Teniendo este resultado, para los juegos no cooperativos se tiene el siguiente lema, que caracteriza los puntos de equilibrio como puntos fijos de la correspondencia de mejor respuesta conjunta.

Lema 2.13. El conjunto de puntos de equilibrio de un juego no cooperativo $\left(N, S,\left\{R_{i}\right.\right.$ : $i \in N\}$ ) es idéntico al conjunto de puntos fijos de la correspondencia de mejor respuesta conjunta $Y(\mathbf{x}), \mathbf{x} \in S$.

\section{DEMOSTRACIÓN}

Se probará que los conjuntos son iguales.

Sea $\mathbf{x} \in S$ un punto fijo de la correspondencia de mejor respuesta conjunta. Considere al jugador $i$ y $y_{i} \in S_{i}\left(x_{-i}\right)$, se tiene que $\mathbf{x}^{\prime}=\left(x_{-i}, y_{i}\right) \in S(\mathbf{x})$, luego,

$$
0 \leq g(\mathbf{x}, \mathbf{x})-g\left(\mathbf{x}^{\prime}, \mathbf{x}\right)=R_{i}(\mathbf{x})-R_{i}\left(x_{-i} ; y_{i}\right)
$$

y esto sucede para cualquier $i \in N$, pues el $i$ elegido fue arbitrario, de aquí que $\mathbf{x}$ sea un punto de equilibrio.

Ahora, tómese $\mathbf{x}$ un punto de equilibrio y $\mathbf{y} \in S(\mathbf{x})$, entonces $y_{i} \in S_{i}\left(x_{-i}\right), \forall i \in N$. Como $\mathbf{x}$ es un punto de equilibrio, $R_{i}(\mathbf{x}) \geq R_{i}\left(x_{-i} ; y_{i}\right)$, luego,

$$
\begin{aligned}
0 \leq \sum_{i \in N}\left(R_{i}(\mathbf{x})-R_{i}\left(x_{-i} ; y_{i}\right)\right) & =\sum_{i \in N} R_{i}(\mathbf{x})-\sum_{i \in N} R_{i}\left(x_{-i} ; y_{i}\right) \\
& =g(\mathbf{x}, \mathbf{x})-g(\mathbf{y}, \mathbf{x})
\end{aligned}
$$

Luego, $g(\mathbf{x}, \mathbf{x}) \geq g(\mathbf{y}, \mathbf{x})$, con $\mathbf{y}$ elegido de manera arbitraria en $S(\mathbf{x})$, así $\mathbf{x}$ maximiza la función $g$, en otras palabras, $\mathbf{x}$ pertenece al conjunto $Y(\mathbf{x})$, es decir, es un punto fijo de la correspondencia de mejor respuesta conjunta.

Contando con lo anterior, si se agregan algunas características al conjunto de perfiles de estrategias y a las funciones de pago, el siguiente resultado nos asegura la existencia de puntos de equilibrio en los juegos supermodulares no cooperativos.

Teorema 2.14. Dado un juego supermodular no cooperativo, $\left(N, S,\left\{R_{i}: i \in N\right\}\right)$, con conjunto de perfiles de estrategias admisibles $S$, no vacío y compacto, cuya función de pago $R_{i}\left(x_{-i} ; y_{i}\right)$ es semicontinua superiormente en $y_{i} \in S_{i}\left(x_{-i}\right)$, para $x_{-i} \in S_{-i}, \forall i \in N$. Entonces, el conjunto de puntos de equilibrio es una latiz completa y no vacía, que tiene elementos máximo y mínimo. 


\section{DEmostración}

Recuerde que el conjunto de puntos de equilibrio de un juego no cooperativo es igual al conjunto de puntos fijos de la correspondencia de mejor respuesta conjunta.

Ahora, observe que:

- $S \subset \mathbb{R}^{m}$ es una sublatiz no vacía y compacta, así, por el teorema $1.31, S$ es una sublatiz subcompleta. De esto que $S$ sea una latiz completa.

- Del lema 2.11, se tiene que la correspondencia de mejor respuesta conjunta es una sublatiz compacta de $\mathbb{R}^{m}$ así, $Y(\mathbf{x})$ es una sublatiz subcompleta.

Teniendo estas observaciones, por el teorema 1.41, se sigue que el conjunto de puntos de equilibrio de la correspondencia de mejor respuesta conjunta es una latiz no vacía y completa, de la completez de este conjunto, se puede concluir que tiene elementos máximo y mínimo.

\subsection{Algoritmos}

En esta sección, se enunciarán dos algoritmos que permiten, dado un juego supermodular no cooperativo, generar sucesiones de perfiles de estrategias admisibles. Después, se presentan resultados que garantizan que dichos algoritmos generan puntos de equilibrio al agregar algunas hipótesis a los conjuntos de estrategias admisibles y a las funciones de utilidad.

\subsubsection{Optimización Round-Robin}

Dado un juego no cooperativo $\left(N, S,\left\{R_{i}: i \in N\right\}\right)$, siguiendo los pasos que se enuncian a continuación, se genera una sucesión (finita o infinita) de perfiles de estrategias admisibles.

a) Si $S$ tiene un elemento mínimo, $\inf (S)$, haga $x^{0,0}=\inf (S)$. En caso contrario, se detiene.

b) Dado $x^{k, i} \in S$, con $k, i \in \mathbb{N}$ y $i<n$, considere $x^{k, i+1}=\left(x_{i+1}^{k, i+1}, x_{-(i+1)}^{k, i}\right)$, donde $x_{i+1}^{k, i+1}$ es el elemento mínimo de la correspondencia de mejor respuesta $Y_{i+1}\left(x_{-(i+1)}^{k, i}\right)$, si este elemento existe. En otro caso, deténgase. 
c) Se incrementa $i=i+1$, si $i=n$ y $x^{k, n}$ se ha generado para algún $k$, considere $x^{k+1,0}=x^{k, n}$, aumente $k=k+1$ y haga $i=0$. Regrese al paso b).

Los siguientes resultados, aseguran que el algoritmo de Round-Robin genera equilibrios en juegos supermodulares no cooperativos e incluso proveen una cota para el número de iteraciones necesarias para encontrar un equilibrio cuando el conjunto de perfiles de estrategias admisibles es finito.

Teorema 2.15. Dado un juego supermodular $\left(N, S,\left\{R_{i}: i \in N\right\}\right)$, con conjunto de perfiles de estrategias admisibles no vacío y compacto, cuyas funciones de utilidad, $R_{i}\left(x_{-i} ; y_{i}\right)$, son semicontinuas superiormente en $y_{i} \in S_{i}\left(x_{-i}\right)$, para cada $x_{-i} \in S_{-i}$. Se tiene que:

a) El algoritmo de Round-Robin no se detiene en los pasos a) o b). Además, genera una sucesión infinita $x^{k, i}$, creciente en $k$ e $i$, para $k=0,1, \ldots, i=0, \ldots, n$. De esto que exista un perfil de estrategias admisibles $x^{*} \in S$ tal que, $\lim _{k \rightarrow \infty} x^{k, i}=$ $x^{*}$, para $i=0, \ldots, n$.

b) Si en la sucesión generada por el algoritmo aparece un perfil de estrategias $n$ veces consecutivas, entonces ese perfil es un punto de equilibrio.

c) Si en alguna iteración se genera un punto de equilibrio, el algoritmo generará el mismo punto en cualquier iteración posterior.

d) Si además, el conjunto de perfiles de estrategias es finito y para cada conjunto de estrategias admisibles $, S_{i}, q_{i}$ es una cota superior de la cardinalidad de cualquier cadena contenida en dicho conjunto, entonces el algoritmo genera un punto de equilibrio en a lo más $(n-1)\left(\sum_{i=1}^{n} q_{i}\right)-n^{2}+n+1$ iteraciones.

La demostración del teorema anterior se puede revisar en [10] (página 187, teorema 4.3.1).

\subsubsection{Optimización simultánea}

Dado un juego no cooperativo $\left(N, S,\left\{R_{i}: i \in N\right\}\right)$, se genera una sucesión (finita o infinita) de perfiles de estrategias admisibles de la siguiente manera.

a) Si $S$ tiene un elemento mínimo, fije $x^{0}=\operatorname{lnf}(S)$. En otro caso, detenerse.

b) Dado $x^{k} \in S$, para cualquier $k \in \mathbb{N}$, tómese como $x^{k+1}$ al mínimo elemento de la correspondencia de mejor respuesta conjunta $Y\left(x^{k}\right)$, si dicho elemento existe. De lo contrario, se detiene el algoritmo. 
c) Haga $k=k+1$. Regrese al paso b).

De manera análoga al algoritmo de Round-Robin, a continuación se presentan resultados que aseguran que el algoritmo de optimización simultánea genera puntos de equilibrio y dan una cota del número de iteraciones necesarias para obtenerlo cuando el conjunto de perfiles de estrategias admisibles es finito.

Teorema 2.16. Considere un juego supermodular $\left(N, S,\left\{R_{i}: i \in N\right\}\right)$, tal que, su conjunto de perfiles de estrategias, $S \neq \emptyset$, sea compacto, y cuyas funciones de utilidad $R_{i}\left(x_{-i} ; y_{i}\right)$ son semicontinuas superiormente en $y_{i} \in S_{i}\left(x_{-i}\right)$, para $x_{-i} \in S_{-i}$. Entonces,

a) El algoritmo de optimización simultánea no se detiene y genera una sucesión $x^{k}$, creciente en $k$, para $k=0,1, \ldots$ De esto que, exista un perfil de estrategias admisibles $x^{*} \in S$, tal que $\lim _{k \rightarrow \infty} x^{k}=x^{*}$.

b) Si un perfil de estrategias admisibles aparece dos veces sucesivas en la sucesión generada con el algoritmo, entonces es un punto de equilibrio.

c) Si el algoritmo genera un punto de equilibrio, entonces ese punto se seguirá generando en cualquier iteración posterior.

d) Si a las hipótesis iniciales se agrega que, el conjunto de perfiles de estrategias admisibles sea finito y $q$ sea una cota para cualquier cadena contenida en este, entonces el algoritmo genera un equilibrio en no más de $q-1$ iteraciones.

La prueba del teorema anterior se encuentra en [10] (página 191, teorema 4.3.3).

\subsection{Conclusiones}

En este capítulo, se definieron los juegos supermodulares no cooperativos. Además, se enunciaron algunos resultados que aseguran la existencia de puntos de equilibrio, bajo ciertas suposiciones sobre el conjunto de estrategias y las funciones de pago, finalmente se presentaron dos algoritmos para encontrar sucesiones de perfiles de estrategias admisibles.

Dicha teoría será empleada en el capítulo 3, en el cual se plantearán algunos ejemplos de juegos supermodulares no cooperativos, de los cuales se procederá a aproximar sus equilibrios implementando los algoritmos dados. 


\section{Capítulo 3}

\section{Aplicaciones}

\subsection{Introducción}

En este capítulo se presentarán algunos ejemplos de juegos supermodulares no cooperativos, en los que es posible aplicar la teoría revisada y aproximar equilibrios mediante los algoritmos descritos en el capítulo 2.

Inicialmente, se expondrán ciertos ejemplos donde se ha hecho uso de los juegos supermodulares no cooperativos, algunos de estos pueden encontrarse en [1], [4], [5], [10], [12], [13].

Posteriormente, se abordará el modelo de productos parcialmente complementarios [2], se presentarán algunos ejemplos numéricos y se aproximarán sus equilibrios usando los programas que se muestran en el apéndice C, los cuales están basados en los algoritmos previamente enunciados.

\subsection{Antecedentes}

\subsubsection{Producción con bienes complementarios [10]}

\section{Planteamiento del modelo}

Sea $N=\{1, \ldots, n\}$, un conjunto de empresas, cada empresa $i$ produce $\nu_{i}$ tipos de bienes, estos son considerados como un único producto, se considera la producción de la empresa como paquetes. 
Se supone que las firmas tomarán decisiones distintas dependiendo del periodo de tiempo $\tau$ en el que se encuentren. Cada firma decide:

- $\nu_{i}$ : cuántos tipos de bienes produce.

- $q_{i}$ : la calidad de sus productos.

- $\theta_{i}$ : nivel de tecnología usada en el proceso de producción.

- $\rho_{i}$ : gastos de publicidad.

- $\sigma_{i}$ : tamaño del mercado al que se presenta el producto.

Así, la estrategia de cada firma está dada por el vector $x_{i}=\left(\nu_{i}, q_{i}, \theta_{i}, \rho_{i}, \sigma_{i}\right)$ y este pertenece a un conjunto de estrategias admisibles $S_{i} \subset \mathbb{R}^{5}$.

\section{Características}

Un perfil de estrategias es de la forma $\mathbf{x}=\left(x_{1}, \ldots, x_{n}\right) \in \prod_{i=1}^{n} S_{i}$.

El precio de cada producto, $p_{i}$, es fijado por el mercado, independientemente de las decisiones de cada firma.

La demanda para la firma $i$, está dada por $\mu_{i}(x, \tau)$ y cada firma produce únicamente para satisfacer esta. Para cada empresa, se deben considerar los siguientes costos:

- Costo de producción de $z$ paquetes de bienes por empresa, dado por $c_{i}\left(z, x_{i}, \tau\right)$; este sólo depende de $\nu_{i}, q_{i}, \theta_{i}$.

- Costo por la tecnología usada, independientemente de los paquetes producidos, dada por $k_{i}\left(x_{i}, \tau\right)$; este costo sólo depende de $\nu_{i}, q_{i}, \theta_{i}$.

- Costo de publicidad, $\rho_{i}$.

- Costo por introducir los productos al mercado, $m_{i}\left(\sigma_{i}, \tau\right)$.

Con estas especificaciones, la función de utilidad es dada por

$$
p_{i} \mu_{i}(\mathbf{x}, \tau)-c_{i}\left(\mu_{i}(\mathbf{x}, \tau), x_{i}, \tau\right)-k_{i}\left(x_{i}, \tau\right)-\rho_{i}-m_{i}\left(\sigma_{i}, \tau\right) .
$$

Si, además:

- los conjuntos de estrategias admisibles de cada jugador, $S_{i}$, son sublatices; 
- las demandas $\mu_{i}$ son crecientes en $\mathbf{x}$, es decir, a mayor inversión de las firmas en su producto, mayor demanda de este;

- las demandas son supermodulares en $x_{i}$ y tienen diferencias crecientes en $\left(x_{i}, x_{-i}\right)$;

- los costos de producción $c_{i}\left(z, x_{i}, \tau\right)$ son cóncavos en $z$ y submodulares en $\left(z, x_{i}\right)$,es decir, $-c_{i}$ es una función supermodular con diferencias crecientes;

- $p_{i} z-c_{i}\left(z, x_{i}, \tau\right)$ es creciente en $z$;

- $k_{i}\left(x_{i}, \tau\right)$ es submodular,

entonces, se tiene que la función (3.1) es supermodular y tiene diferencias crecientes, por ende, el modelo se puede ver como un juego supermodular no cooperativo.

En el juego descrito, los equilibrios representan las decisiones que deben tomar las firmas, respecto a la inversión que harán en sus productos, esta incluye, cuantos tipos de bienes producir, cuanto deben invertir en tecnología y publicidad, la calidad de su producto y el mercado en el cual lo van a ofertar, esto para maximizar sus ganancias, cuando las estrategias de las demás firmas están fijas en dicho punto.

\subsubsection{Redes AdHoc inalámbricas [5]}

\section{Planteamiento del modelo}

El término red AdHoc se refiere a las redes en las que todos los nodos tienen el mismo estado en la red, es decir, cada nodo toma sus decisiones independientemente, o en otras palabras, la red carece de control central.

Considere $N=\{1, \ldots, n\}$ pares de nodos distintos, cada usuario (link), consiste de un transmisor y un receptor. La potencia transmitida y $\mathrm{SINR}^{1}$ del $i$-ésimo usuario se denotan por $p_{i}$ y $\gamma_{i}$, respectivamente; la potencia transmitida es un valor en $\mathbb{R}$, tal que $p_{i} \in\left[p_{\text {mín }}^{i}, p_{\text {máx }}^{i}\right]$. El vector de potencia transmitida por los usuarios está dado por $\mathbf{p}=\left(p_{1}, \ldots, p_{n}\right) \in S \subset \mathbb{R}^{n}$ y el ruido en las señales recibidas en el ancho de banda del usuario $i$ es $\sigma_{i}$.

El objetivo en el control de potencia en redes inalámbricas es asegurar que el SINR de ningún usuario, $\gamma_{i}$, sea menor que el umbral requerido $\gamma_{i}^{*}$ elegido para asegurar la QoS ${ }^{2}$ adecuada.

\footnotetext{
${ }^{1}$ Relación señal a interferencia más ruido

${ }^{2}$ Calidad de servicio
} 


\section{Características}

En este problema, el SINR es

$$
\gamma_{i}(\mathbf{p})=\frac{p_{i} h_{i i}}{\sigma_{i}+\sum_{j \neq i} p_{j} h_{j i}}
$$

con $h_{j i}$ la ganancia del transmisor $T_{j}$, del usuario $j$, al receptor del usuario $i$ y donde el denominador de (3.2) es la interferencia más ruido del usuario $i$.

Las aplicaciones de datos, son sensibles al error pero, tolerantes al retraso. Un mayor nivel de SINR en la salida del usuario receptor resultará en una menor tasa de error y, por ende, en mayor rendimiento. Sin embargo, para conseguir niveles de SINR altos se requiere transmitir a mayor potencia, lo cual resulta en reducción del tiempo de vida de la bateria. Además, mayor potencia incrementa la magnitud de la interferencia para los usuarios restantes. Esto se puede cuantificar definiendo la función de utilidad de la cantidad promedio de datos recibidos correctamente por unidad de energía consumida como

$$
U_{i}(\mathbf{p})= \begin{cases}\frac{T_{i}(\mathbf{p})}{p_{i}}, & \text { si } \gamma_{i}(\mathbf{p}) \geq \gamma_{i}^{*} \\ 0, & \text { en otro caso }\end{cases}
$$

Se supone que los transmisores usan una tasa variable de M-QAM ${ }^{3}$ con probabilidad acotada de error de símbolo y codificación de trellis ${ }^{4}$ con ganancia de codificación nominal, estas suposiciones implican que la función de utilidad es supermodular. El rendimiento efectivo se puede aproximar por $T_{i}(\mathbf{p})=W \log _{2}\left(1+\frac{\gamma_{i}(\mathbf{p})}{\Omega}\right)$, que es función del vector potencia global y las condiciones del canal, donde

- W, representa el ancho de banda,

- $\Omega$, es la diferencia entre el M-QAM y la capacidad menos la ganancia de codificación.

Este es un juego supermodular no cooperativo, en el cual, un equilibrio representa la potencia que debe transmitir cada usuario para maximizar la cantidad promedio de datos recibidos correctamente por unidad de energía consumida, cuando los demás usuarios fijan su potencia a transmitir en dicho punto.

\footnotetext{
${ }^{3}$ Modulación de amplitud en cuadratura

${ }^{4}$ Modulación con codificación reticulada
} 


\subsubsection{Fijación de precios con productos reemplazables [1], $[10]$}

\section{Planteamiento del modelo}

Sea $N=\{1, \ldots, n\}$ un conjunto de firmas, cada una de ellas produce un bien y los $n$ productos pueden ser sustituidos entre sí. Cada firma $i$ fija el precio de su producto, denote dicho precio por $x_{i}$.

\section{Características}

El vector de precios $\mathbf{x}=\left(x_{1}, \ldots, x_{n}\right)$ pertenece a $S \subseteq \mathbb{R}^{n}$. Los precios de venta no pueden ser menores que el costo de producción, dado por $t_{i} \geq 0$, así $S \subseteq \Pi_{i=1}^{n}\left[t_{i}, \infty\right)$. Además, el precio de cada bien no puede superar una cota, $a_{i}$, fijada en base a la calidad del bien y la cantidad máxima que el público está dispuesto a pagar por ese producto. Así, los vectores de precios admisibles son elementos del conjunto $S=\prod_{i=1}^{n}\left[t_{i}, a_{i}\right]$. Este es el conjunto de perfiles de estrategias admisibles del juego

La demanda de cada producto $i$, está dada por una función $D_{i}$, dependiente del vector de precios y, cada firma produce únicamente la cantidad de bienes demandados.

Las funciones de demanda son crecientes, para $i, j$ distintos, es decir, si el precio del bien $j$ aumenta, entonces también lo hará la demanda del $i$-ésimo. Además, las demandas tienen diferencias crecientes, pues, para $x_{j}<x^{\prime}{ }_{j} \mathrm{y} x_{-j}<x_{-j}^{\prime}, D_{i}\left(x_{-j}^{\prime}, x_{j}\right)-$ $D_{i}\left(x_{-j}, x_{j}\right) \leq D_{i}\left(x^{\prime}{ }_{-j}, x^{\prime}{ }_{j}\right)-D_{i}\left(x_{-j}, x^{\prime}{ }_{j}\right)$, en otras palabras, la demanda de un producto es más sensible a su precio si los precios de los demás productos son menores.

Cada empresa recibe $x_{i} D_{i}(\mathbf{x})$ por su producción. Así, las utilidades de cada una están dadas por

$$
R_{i}(\mathbf{x})=\left(x_{i}-t_{i}\right) D_{i}(\mathbf{x})
$$

En este modelo, los equilibrios son los precios que debe fijar cada empresa para maximimzar su demanda, por ende sus utilidades, cuando se mantienen los precios de las otras firmas en dicho punto. 


\subsubsection{Oligopolios multimercado [10], [12]}

\section{Planteamiento del modelo}

Sea $N=\{1, \ldots, n\}$, un conjunto de firmas involucradas en actividades de $m$ mercados. En el mercado $j$, la firma $i$ tiene un vector de nivel de actividad $x_{i, j} \in$ $S_{i, j} \in \mathbb{R}^{k_{i, j}}$, donde $k_{i, j}$ es el tamaño de dicho vector. Las estrategias admisibles de cada firma están dadas por $x_{i}=\left(x_{i, 1}, \ldots, x_{i, m}\right)$; un perfil de estrategias de las $n$ firmas, es de la forma $\mathbf{x}=\left(x_{1}, \ldots, x_{n}\right)$. El conjunto de perfiles de estrategias admisibles es $S=\prod_{i=1}^{n}\left(\prod_{j=1}^{m} S_{i, j}\right)$.

\section{Características}

La función de utilidad de cada firma está dada por

$$
f_{i}(\mathbf{x})=\sum_{j=1}^{m} h_{i, j}\left(x_{1, j}, \ldots, x_{n, j}\right)-c_{i}\left(x_{i}\right),
$$

donde

- $h_{i, j}$ es la ganancia de la $i$-ésima firma por sus actividades en el $j$-ésimo mercado,

- $c_{i}$ es el costo por las estrategias tomadas en todos los mercados que participa la firma $i$.

Este modelo es un juego supermodular no cooperativo cuando los conjuntos de estrategias $S_{i, j}$ son sublatices, los ingresos de cada firma por sus actividades en cada mercado $h_{i, j}$ son supermodulares en $x_{i, j}$ y tienen diferencias crecientes en $\left(x_{i, j}, x_{-i, j}\right)$ y $c_{i}\left(x_{i}\right)$ es submodular.

En este modelo, los equilibrios representan los niveles de actividad de las $n$ firmas, en cada uno de los $m$ mercados, que maximizan su utilidad cuando los niveles de actividad de las demás son los dados en dicho equilibrio.

\subsubsection{Teoría de Colas [13]}

\section{Planteamiento del modelo}

Considere una cola de dos servidores en tándem donde, cada servidor tiene tiempos de servicio i.i.d. exponenciales con tasas $\mu_{1}$ y $\mu_{2}$, respectivamente. Considere una 
cota superior de las tasas de servicio, $u$, tal que $u \geq \mu_{1} \vee \mu_{2}$.

La fuente del servidor 1 se considera infinita, al igual que las capacidades de ambas colas. El rendimiento del sistema, está dado por $\mu_{1} \wedge \mu_{2}$.

Las tasas de servicio admisibles, para ambos servidores están dadas por $S_{1}\left(\mu_{2}\right)=$ $\left\{\mu_{1}: 0 \leq \mu_{1} \leq \mu_{2}\right\}$ y $S_{2}\left(\mu_{1}\right)=\left\{\mu_{2}: \mu_{1} \leq \mu_{2} \leq u\right\}$. Note que estos conjuntos, al estar contenidos en $\mathbb{R}$, son sublatices.

\section{Características}

Para $i=1,2$, sean

- $p_{i}\left(\mu_{1} \wedge \mu_{2}\right)$, la función de beneficio del servidor $i$,

- $c_{i}\left(\mu_{i}\right)$, el costo de operación del servidor $i$,

- $g(\cdot)$, el costo por tener trabajos pendientes en cola.

El objetivo del problema es maximizar las funciones de utilidad dadas por

$$
f_{i}\left(\mu_{1}, \mu_{2}\right)=p_{i}\left(\mu_{1} \wedge \mu_{2}\right)-c_{i}\left(\mu_{i}\right)-g\left(\frac{\mu_{1}}{\mu_{2}-\mu_{1}}\right)
$$

Sean $p_{i}$ y $c_{i}$ continuas y $\lim _{x \rightarrow \infty} g(x)=\infty$. Si $g$ es creciente y convexa, las funciones de utilidad son supermodulares en $\left(\mu_{1}, \mu_{2}\right)$.

En este ejemplo, los equilibrios dan las tasas de servicio que maximizan las utilidades de los servidores.

\subsubsection{Búsqueda de socios comerciales [1], [10]}

\section{Planteamiento del modelo}

Sea $N=\{1, \ldots, n\}$ un grupo de firmas buscando socios comerciales, el nivel de búsqueda de cada firma está dado por $x_{i} \in S_{i} \subset \mathbb{R}$. Un perfil de estrategias de las $n$ firmas está dado por $\mathbf{x}=\left(x_{1}, \ldots, x_{n}\right) \in S$, con $S=\prod_{i=1}^{n} S_{i}$ conjunto de perfiles de estrategias admisibles. 


\section{Características}

La función de utilidad de la firma $i$ está dada por

$$
f_{i}(\mathbf{x})=g_{i}(\mathbf{x})-c_{i}\left(x_{i}\right)
$$

donde

- $c_{i}$ es el costo por el esfuerzo realizado en la búsqueda de socios.

- $g_{i}$ es la ganancia esperada del intercambio comercial.

Considere que las funciones $g_{i}$ tienen diferencias crecientes, esto implica que, si la firma $i$ aumenta su esfuerzo en la búsqueda de socios, se espera que sus ganancias aumenten cuando las demás firmas también invierten más esfuerzo en su búsqueda. Además, si las $S_{i}$ son sublatices compactas; las funciones de $\operatorname{costo} c_{i}$ son semicontinuas inferiormente, tienen diferencias decrecientes y las $g_{i}$ son semicontinuas superiormente, el modelo planteado es un juego supermodular no cooperativo, en el cual, los equilibrios dan el esfuerzo que debe realizar cada firma para maximizar sus ganancias del intercambio comercial sin exceder sus esfuerzos en la búsqueda de socios, cuando las demás firmas tienen fijo su nivel de búsqueda en el equilibrio.

\subsection{Productos parcialmente complementarios}

En un principio, se explicará lo que son los productos parcialmente complementarios, seguido de esto, se proporcionan algunos ejemplos de este tipo de productos.

Se concluirá el apartado, mostrando algunos ejemplos numéricos en los cuales se aproximarán los equilibrios y se explicará que quieren decir estos en el contexto de dichos ejemplos.

El modelo planteado en este apartado fue encontrado en el artículo de Xiang Fang, Capacity Games for Partially Complementary Products Under Multivariate Random Demands [2], en dicho artículo, se propone el modelo de productos parcialmente complementarios como un juego no cooperativo y se esboza como encontrar equilibrios para este resolviendo un sistema de ecuaciones diferenciales.

En esta tesis se muestra que, para algunas distribuciones, bajo ciertas condiciones, las funciones de utilidad son supermodulares, esto y algunas observaciones sobre los conjuntos de estrategias admisibles, permiten plantear el modelo como un juego 
supermodular no cooperativo, con ello, obtener los equilibrios del juego se puede lograr aplicando los algoritmos descritos en el capítulo anterior.

Se dice que un conjunto de bienes son parcialmente complementarios si estos se pueden solicitar de manera conjunta o individualmente, en otras palabras, son aquellos productos que enfrentan demandas comunes e individuales.

Ejemplo 3.1. Los siguientes son productos parcialmente complementarios:

1. monitores y teclados,

2. llantas, motores y demás autopartes,

3. materiales de construcción, ladrillos, cemento, etc.

\subsubsection{Planteamiento del modelo}

Se consideran $n$ firmas, las cuales producen bienes parcialmente complementarios. La demanda común de las firmas es denotada por $D_{0}$, dicha demanda solo se puede cumplir cuando todas las firmas tienen inventario. Además, cada firma tiene su propio mercado, independiente de las demás, cuya demanda está dada por $D_{i}$, $i=1, \ldots, n$. Las demandas, $D_{0}, D_{1}, \ldots, D_{n}$, son desconocidas y siguen una distribución multivariada continua.

Cada firma debe elegir su producción, $Q_{i}$, con la finalidad de maximizar sus ganancias y, su decisión debe ser tomada antes de conocer las demandas de sus bienes. Una vez conocidas las demandas, cada firma $i$, debe elegir como distribuir su producto entre las demandas $D_{0}$ y $D_{i}$, esta asignación, dependerá del precio de venta por cada demanda, a manera de darle prioridad a la demanda con precio mayor. Las demandas que no son satisfechas se consideran perdidas.

Las relaciones posibles entre los precios de venta individual y conjunta, $p_{i}$ y $p_{0 i}$, se muestran en los casos dados a continuación:

a) $p_{i}>p_{0 i}$, para $i=1, \ldots, n$. Es decir, el precio de venta individual de cada firma es mayor que el de ventaa conjunta, lo cual nos dice que cada empresa abastecerá su demanda individual y posteriormente intentará surtir la demanda común. 
b) $p_{i} \leq p_{0 i}$, para $i=1 \ldots, n$. En este caso, todas las firmas buscarán llenar la demanda común y posteriormente, su respectiva demanda individual.

c) $p_{i} \leq p_{0 i}$ para algunas firmas y $p_{i}>p_{0 i}$ para las demás. En esta situación, dependiendo del caso correspondiente, la empresa decidirá cual de sus demandas satisface primero.

La tesis se enfoca en el caso a), en este, cada firma desea llenar su demanda individual en la medida de lo posible y posteriormente, la demanda común.

Como la demanda común requiere que los $n$ productos estén disponibles, cada firma puede vender únicamente $\operatorname{mín}_{i=1 \ldots, n}\left\{D_{0},\left(Q_{i}-D_{i}\right)^{+}\right\}$. Así, la función de utilidad esperada de cada firma está dada por

$$
E \Pi_{i}\left(Q_{i} \mid Q_{-i}\right)=E\left[p_{i} \operatorname{mín}\left\{D_{i}, Q_{i}\right\}+p_{0 i} \operatorname{mín}_{k=1 \ldots, n}\left\{D_{0},\left(Q_{k}-D_{k}\right)^{+}\right\}-c_{i} Q_{i}\right],
$$

donde $c_{i}$ es el costo de producción del bien correspondiente a la firma $i$, y es tal que $c_{i}<\operatorname{máx}\left\{p_{i}, p_{0 i}\right\}$.

Nota 3.2. Se considera el caso en el que todas las demandas son independientes, un ejemplo en el que sucedería esto es si la demanda común se tiene en un mercado y las demandas individuales se tienen en otro y estos no están relacionados.

\subsubsection{Demandas distribuidas uniformemente}

Para este apartado, considere dos firmas con productos parcialmente complementarios cuyas demandas, individuales y conjunta, son independientes y se distribuyen uniformemente en un intervalo $[a, b]$.

Con estos supuestos, la función de utilidad esperada, dada en (3.4), queda de la 
siguiente manera:

$$
\begin{aligned}
E \Pi_{i}\left(Q_{i} \mid Q_{k}\right)= & p_{i} Q_{i} P\left(Q_{i}<D_{i}\right)+\frac{p_{i}}{b-a} \int_{a}^{\alpha_{i}} y d y-c_{i} Q_{i} \\
& +\frac{p_{0 i}}{b-a}\left\{\int_{a}^{b} y P\left(D_{i}<Q_{i}-y\right) P\left(D_{k}<Q_{k}-y\right) d y\right. \\
& +Q_{i} \int_{a}^{\alpha_{i}} P\left(D_{0}>Q_{i}-y\right) P\left(D_{k}<Q_{k}+y-Q_{i}\right) d y \\
& -\int_{a}^{\alpha_{i}} y P\left(D_{0}>Q_{i}-y\right) P\left(D_{k}<Q_{k}+y-Q_{i}\right) d y \\
& +Q_{k} \int_{a}^{\alpha_{k}} P\left(D_{0}>Q_{k}-y\right) P\left(D_{i}<Q_{i}+y-Q_{k}\right) d y \\
& \left.-\int_{a}^{\alpha_{k}} y P\left(D_{0}>Q_{k}-y\right) P\left(D_{i}<Q_{i}+y-Q_{k}\right) d y\right\},
\end{aligned}
$$

donde $\alpha_{\ell}=\operatorname{mín}\left\{b, Q_{\ell}\right\}$, para $\ell=i, k$; ver (B.3) en apéndice B.

Dependiendo del valor que tome $\alpha_{\ell}$, la función de utilidad tiene cuatro casos posibles (para más detalles ver apéndice B), considere el caso en el que $\alpha_{\ell}=Q_{\ell}$, para $\ell=i, k$, es decir, la producción de cada firma no rebasa la cota superior de ninguna de las demandas. En este caso, la función de utilidad tiene segunda derivada no negativa y por ende diferencias crecientes, luego, por el teorema 1.53, es supermodular.

Así, para demandas distribuidas uniformemente, independientes, con producciones que no exceden la cota superior de las demandas, el modelo de productos parcialmente complementarios se puede ver como un juego supermodular no cooperativo.

Contando con lo anterior, si se consideran los conjuntos de estrategias admisibles de cada firma, es decir, las posibles producciones, como intervalos $[c, d] \subset[a, b]$, se tiene que en el juego supermodular no cooperativo descrito por los productos parcialmente complementarios, se pueden aproximar equilibrios usando los algoritmos de optimización Round-Robin y simultánea, dados en el capítulo anterior.

\subsubsection{Ejemplos}

A continuación, se muestran algunos ejemplos numéricos del modelo de productos parcialmente complementarios y los equilibrios encontrados en estos usando los códigos dados en el apéndice C, que implementan el algoritmo de optimización Round- 
Robin. En dichos ejemplos, los equilibrios representan el nivel de producción que debería tener cada firma para maximizar sus utilidades.

\section{Ejemplo 1}

Considere dos firmas que producen bienes parcialmente complementarios, cuyas demandas, $D_{0}, D_{1}$, y $D_{2}$ se distribuyen uniformente en $[0,1000]$. Cada firma con conjunto de estrategias admisibles dado por $[1,999]$. Cuyos precios de venta y costos de producción están dados en la siguiente tabla:

\begin{tabular}{|l|c|c|}
\hline & Firma 1 & Firma 2 \\
\hline \hline Precio de venta demanda individual & 5 & 6 \\
Precio de venta demanda conjunta & 3 & 4 \\
Costo de producción & 3 & 2 \\
\hline
\end{tabular}

Cuadro 3.1: Ejemplo 1. Precios de venta y costos.

Con estos datos, las funciones de utilidad esperada de cada firma son:

$$
\begin{aligned}
E \Pi_{1}\left(Q_{1} \mid Q_{2}\right)= & 750+Q_{1}-\frac{Q_{1}{ }^{2}}{400}-\frac{3 Q_{1}{ }^{4}}{4000000000}-Q_{2}+\frac{3 Q_{1} Q_{2}}{2000} \\
& +\frac{3 Q_{1}{ }^{2} Q_{2}}{2000000}-\frac{Q_{1}^{3} Q_{2}}{1000000000}+\frac{3 Q_{1} Q_{2}{ }^{2}}{2000000}-\frac{Q_{2}{ }^{3}}{1000000} \\
& -\frac{Q_{1} Q_{2}{ }^{3}}{1000000000}+\frac{3 Q_{2}{ }^{4}}{4000000000} \\
E \Pi_{2}\left(Q_{2} \mid Q_{1}\right)= & 1000+\frac{8 Q_{2}}{3}-\frac{3 Q_{2}}{1000}-\frac{Q_{2}{ }^{3}}{750000}+\frac{Q_{2}{ }^{4}}{1000000000} \\
& -\frac{4 Q_{1}}{3}+\frac{Q_{1} Q_{2}}{500}+\frac{Q_{1} Q_{2}{ }^{2}}{500000}-\frac{Q_{1} Q_{2}{ }^{3}}{750000000}+\frac{Q_{1}{ }^{2} Q_{2}}{500000} \\
& -\frac{Q_{1}{ }^{2}}{750000}-\frac{Q_{1}{ }^{3} Q_{2}}{750000000}+\frac{Q_{1}{ }^{4}}{1000000000}
\end{aligned}
$$

Los perfiles de estrategias obtenidos usando el algoritmo de optimización RoundRobin, se muestran en el cuadro 3.2. 
Observe que las iteraciones posteriores a la 14 generan el mismo perfil de estrategias lo cual, por el teorema 2.15, asegura que el perfil obtenido en dicha iteración es el equilibrio de Nash buscado.

Cuadro 3.2: Perfiles de estrategias admisibles, ejemplo 1.
\begin{tabular}{c|c|c} 
Número de iteración & Jugador $\mathbf{1}$ & Jugador 2 \\
\hline 0 & 1.0000 & 1.0000 \\
1 & 183.8400 & 468.3764 \\
2 & 396.1432 & 584.4921 \\
3 & 456.8978 & 619.5716 \\
4 & 475.0846 & 630.1228 \\
5 & 480.5208 & 633.2782 \\
6 & 482.1430 & 634.2198 \\
7 & 482.6268 & 634.5006 \\
8 & 482.7711 & 634.5843 \\
9 & 482.8141 & 634.6093 \\
10 & 482.8269 & 634.6167 \\
11 & 482.8307 & 634.6189 \\
12 & 482.8318 & 634.6196 \\
13 & 482.8322 & 634.6198 \\
\hline $\mathbf{1 4}$ & $\mathbf{4 8 2 . 8 3 2 3}$ & $\mathbf{6 3 4 . 6 1 9 9}$ \\
15 & 482.8323 & 634.6199 \\
16 & 482.8323 & 634.6199 \\
17 & 482.8323 & 634.6199 \\
\hline
\end{tabular}

Las figuras 3.1 y 3.2 muestran la sucesión creciente de perfiles de estrategias obtenidos usando el algoritmo de Round-Robin.

Las utilidades esperadas para cada firma, dado el perfil de estrategias obtenido, $\left(Q_{1}, Q_{2}\right)=(482.8323,634.6199)$, son:

$$
E \Pi_{1}\left(Q_{1}, Q_{2}\right)=588 \quad E \Pi_{2}\left(Q_{1}, Q_{2}\right)=1603.9
$$

\section{Ejemplo 2}

Considere dos firmas que producen bienes parcialmente complementarios con demandas, $D_{0}, D_{1}$ y $D_{2}$ distribuidas en $[0,8000]$, los posibles niveles de producción de cada empresa están dados por el intervalo [1,7999]. A continuación, se muestran los precios de venta y costos de producción de cada empresa. 


\begin{tabular}{|l|c|c|}
\hline & Firma 1 & Firma 2 \\
\hline \hline Precio de venta demanda individual & 10 & 9 \\
Precio de venta demanda conjunta & 8 & 7 \\
Costo de producción & 5 & 6 \\
\hline
\end{tabular}

Cuadro 3.3: Ejemplo 2. Precios de venta y costos.

Las funciones de utilidad esperada para cada firma están dadas por:

$$
\begin{aligned}
E \Pi_{1}\left(Q_{1} \mid Q_{2}\right)= & 16000+\frac{7 Q_{1}}{3}-\frac{Q_{1}{ }^{2}}{1600}-\frac{Q_{1}{ }^{3}}{24000000}+\frac{Q_{1}{ }^{4}}{256000000000} \\
& -\frac{8 Q_{2}}{3}+\frac{Q_{1} Q_{2}}{2000}+\frac{Q_{2} Q_{1}{ }^{2}}{16000000}-\frac{Q_{2} Q_{1}{ }^{3}}{192000000000} \\
& +\frac{Q_{1} Q_{2}{ }^{2}}{16000000}+\frac{Q_{2}{ }^{3}}{24000000}-\frac{Q_{1} Q_{2}{ }^{3}}{192000000000} \\
& +\frac{Q_{2}{ }^{4}}{256000000000} \\
E \Pi_{2}\left(Q_{2} \mid Q_{1}\right)= & 14000+\frac{2 Q_{2}}{3}-\frac{9 Q_{2}{ }^{2}}{16000}-\frac{7 Q_{2}{ }^{3}}{192000000}+\frac{7 Q_{2}{ }^{4}}{2048000000000} \\
& -\frac{7 Q_{2}}{3}+\frac{7 Q_{1} Q_{2}}{16000}+\frac{7 Q_{1} Q_{2}{ }^{2}}{128000000}-\frac{7 Q_{1} Q_{2}{ }^{3}}{1536000000000} \\
& +\frac{7 Q_{2} Q_{1}{ }^{2}}{128000000}+\frac{7 Q_{1}{ }^{3}}{192000000}-\frac{7 Q_{2} Q_{1}{ }^{3}}{1536000000000} \\
& +\frac{7 Q_{1}^{4}}{2048000000000}
\end{aligned}
$$

Los perfiles de estrategias obtenidos usando el algoritmo de optimización de Round-Robin se muestran en el cuadro 3.4.

El perfil de estrategias encontrado en la iteración 13 del algoritmo se repite en las iteraciones posteriores, de esto que sea el perfil deseado, así, para este ejemplo, el equilibrio está dado por $\left(Q_{1}, Q_{2}\right)=(2728.24,2035.36)$. Con estas estrategias, las utilidades esperadas de cada firma son:

$$
E \Pi_{1}\left(Q_{1}, Q_{2}\right)=9665.8 \quad E \Pi_{2}\left(Q_{1}, Q_{2}\right)=9443.8
$$

En las figuras 3.3 y 3.4, se muestran las gráficas de las estrategias obtenidas para cada jugador. 
Cuadro 3.4: Perfiles de estrategias admisibles, ejemplo 2.

\begin{tabular}{c|c|c} 
Número de iteración & Jugador $\mathbf{1}$ & Jugador 2 \\
\hline 0 & 1.0000 & 1.0000 \\
1 & 1650.9 & 1379.04 \\
2 & 2342.78 & 1792.77 \\
3 & 2582.38 & 1942.74 \\
4 & 2672.12 & 1999.62 \\
5 & 2706.53 & 2021.52 \\
6 & 2719.83 & 2030 \\
7 & 2724.98 & 2033.28 \\
8 & 2726.98 & 2034.56 \\
9 & 2727.76 & 2035.06 \\
10 & 2728.06 & 2035.25 \\
11 & 2728.18 & 2035.33 \\
12 & 2728.23 & 2035.36 \\
\hline $\mathbf{1 3}$ & $\mathbf{2 7 2 8 . 2 4}$ & $\mathbf{2 0 3 5 . 3 6}$ \\
14 & 2728.24 & 2035.36 \\
15 & 2728.24 & 2035.36 \\
16 & 2728.24 & 2035.36 \\
\hline
\end{tabular}

\section{Ejemplo 3}

Suponga dos firmas productoras de bienes parcialmente complementarios, con demandas, común e individuales, distribuidas uniformemente en el intervalo [0, 10000]. Las posibles producciones de cada firma dadas por [1,9999]. Con precios de venta y costos de producción dados por:

\begin{tabular}{|l|c|c|}
\hline & Firma 1 & Firma 2 \\
\hline \hline Precio de venta demanda individual & 16 & 15 \\
Precio de venta demanda conjunta & 12 & 10 \\
Costo de producción & 6 & 8 \\
\hline
\end{tabular}

Cuadro 3.5: Ejemplo 3. Precios de venta y costos.

Con los datos proporcionados, se tiene que las funciones de utilidad para ambas 
firmas están dadas por:

$$
\begin{aligned}
E \Pi_{1}\left(Q_{1} \mid Q_{2}\right)= & 3000+6 Q_{1}-\frac{Q_{1}{ }^{2}}{1250}+\frac{Q_{1}{ }^{3}}{25000000}+\frac{Q_{1}{ }^{4}}{1000000000000}-4 Q_{2} \\
& +\frac{3 Q_{1} Q_{2}}{5000}+\frac{3 Q_{1}{ }^{2} Q_{2}}{50000000}-\frac{Q_{1}{ }^{3} Q_{2}}{250000000000}+\frac{3 Q_{1} Q_{2}{ }^{2}}{50000000} \\
- & \frac{Q_{2}{ }^{3}}{25000000}-\frac{Q_{1} Q_{2}{ }^{3}}{250000000000}+\frac{3 Q_{2}{ }^{4}}{1000000000000} \\
E \Pi_{2}\left(Q_{2} \mid Q_{1}\right)= & 25000+\frac{11 Q_{2}}{3}-\frac{3 Q_{2}{ }^{2}}{4000}-\frac{Q_{2}{ }^{3}}{30000000}+\frac{Q_{2}{ }^{4}}{400000000000} \\
& -\frac{10 Q_{1}}{3}+\frac{Q_{1} Q_{2}}{2000}+\frac{Q_{1} Q_{2}{ }^{2}}{20000000}-\frac{Q_{1} Q_{2}{ }^{3}}{3000000000000} \\
& +\frac{Q_{2} Q_{1}{ }^{2}}{20000000}-\frac{Q_{1}{ }^{3}}{30000000}-\frac{Q_{2} Q_{1}{ }^{3}}{300000000000} \\
& +\frac{Q_{1}{ }^{4}}{400000000000}
\end{aligned}
$$

Usando el algoritmo de optimización Round-Robin, se encontraron los perfiles de estrategias admisibles dados en el cuadro 3.6.

Se concluye que el equilibrio de Nash buscado es $\left(Q_{1}, Q_{2}\right)=(6374.6,5493.6)$ y las utilidades de cada firma, con estas estrategias están dadas por:

$$
E \Pi_{1}\left(Q_{1}, Q_{2}\right)=40487 \quad E \Pi_{2}\left(Q_{1}, Q_{2}\right)=23528
$$

Las figuras 3.5 y 3.6, muestran las gráficas de las estrategias obtenidas.

\section{Gráficas}

A continuación, se muestran las gráficas de las estrategias admisibles generadas mediante el algoritmo de optimización Round-Robin , para cada jugador de los ejemplos recien enunciados. 
Cuadro 3.6: Perfiles de estrategias admisibles, ejemplo 3.

\begin{tabular}{c|c|c} 
Número de iteración & Jugador 1 & Jugador 2 \\
\hline 0 & 1.0000 & 1.0000 \\
1 & 3222.6 & 3714 \\
2 & 5245.5 & 4854.5 \\
3 & 5967.3 & 5265 \\
4 & 6229.2 & 5412.3 \\
5 & 6323 & 5464.8 \\
6 & 6356.3 & 5483.4 \\
7 & 6368.2 & 5490 \\
8 & 6372.4 & 5492.4 \\
9 & 6373.8 & 5493.2 \\
10 & 6374.4 & 5493.5 \\
\hline $\mathbf{1 1}$ & $\mathbf{6 3 7 4 . 6}$ & $\mathbf{5 4 9 3 . 6}$ \\
12 & 6374.6 & 5493.6 \\
13 & 6374.6 & 5493.6 \\
14 & 6374.6 & 5493.6 \\
15 & 6374.6 & 5493.6 \\
\hline
\end{tabular}


Figura 3.1: Ejemplo 1. Estrategias obtenidas, jugador 1.

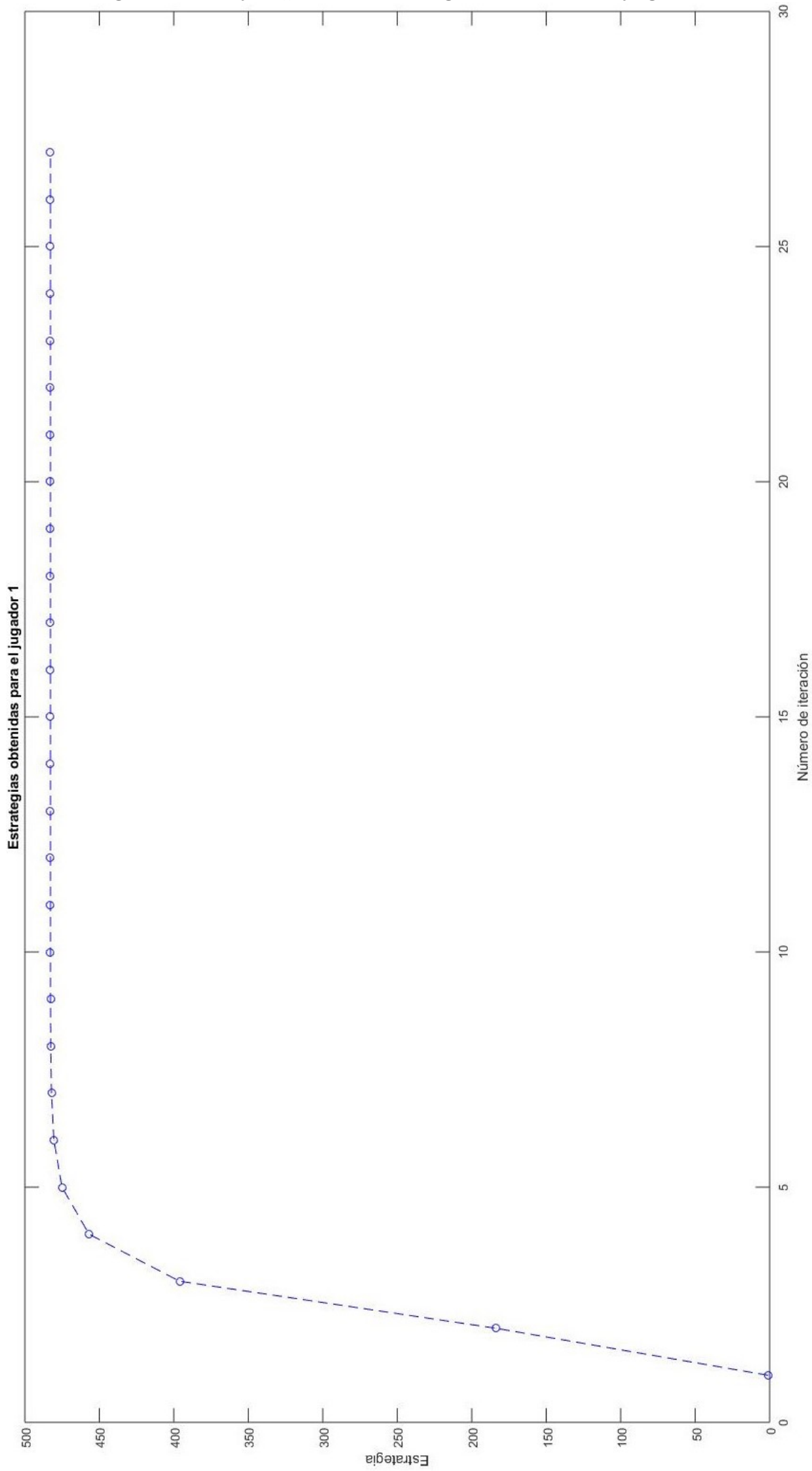


Figura 3.2: Ejemplo 1. Estrategias obtenidas, jugador 2.

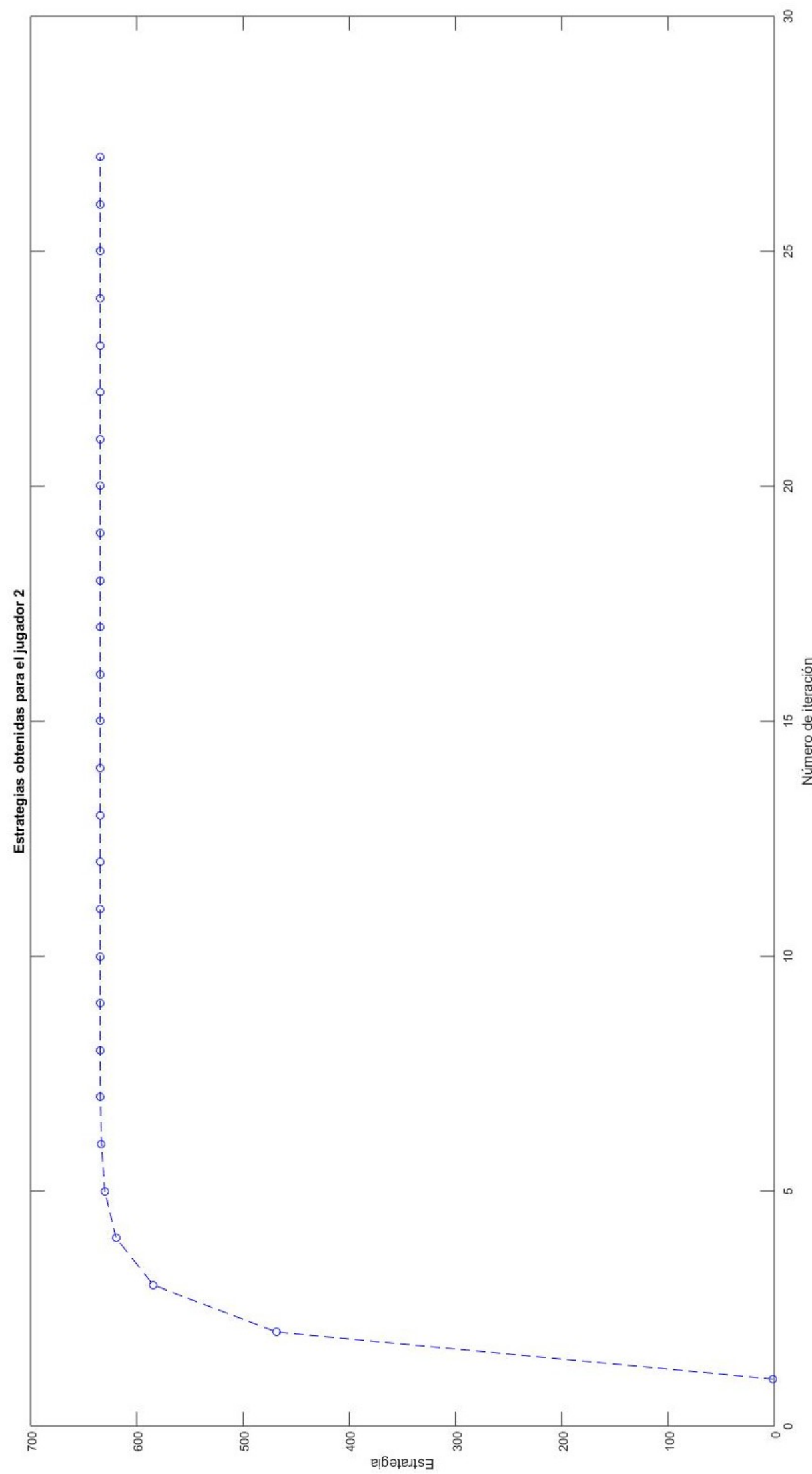


Figura 3.3: Ejemplo 2. Estrategias obtenidas, jugador 1.

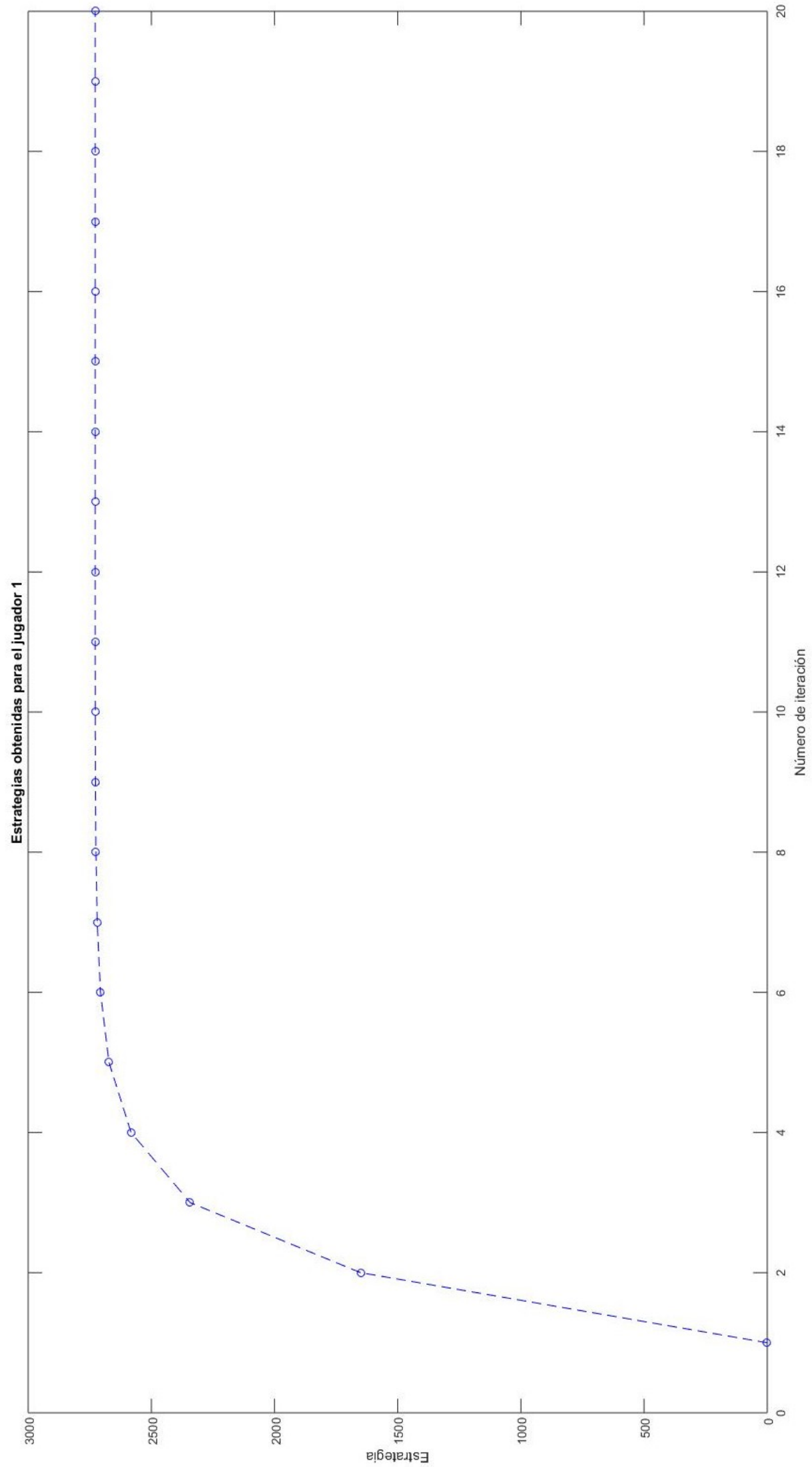


Figura 3.4: Ejemplo 2. Estrategias obtenidas, jugador 2.

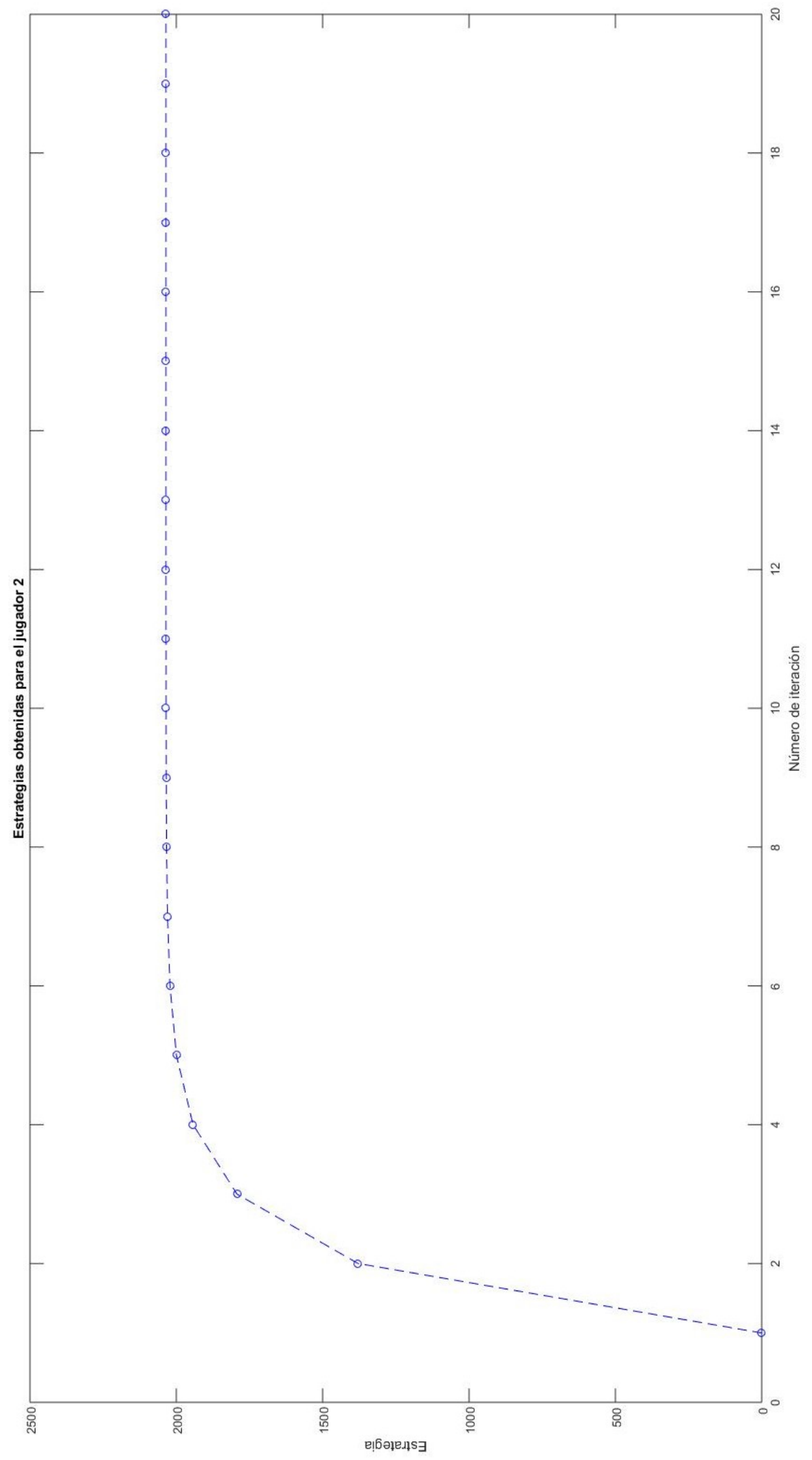


Figura 3.5: Ejemplo 3. Estrategias obtenidas, jugador 1.

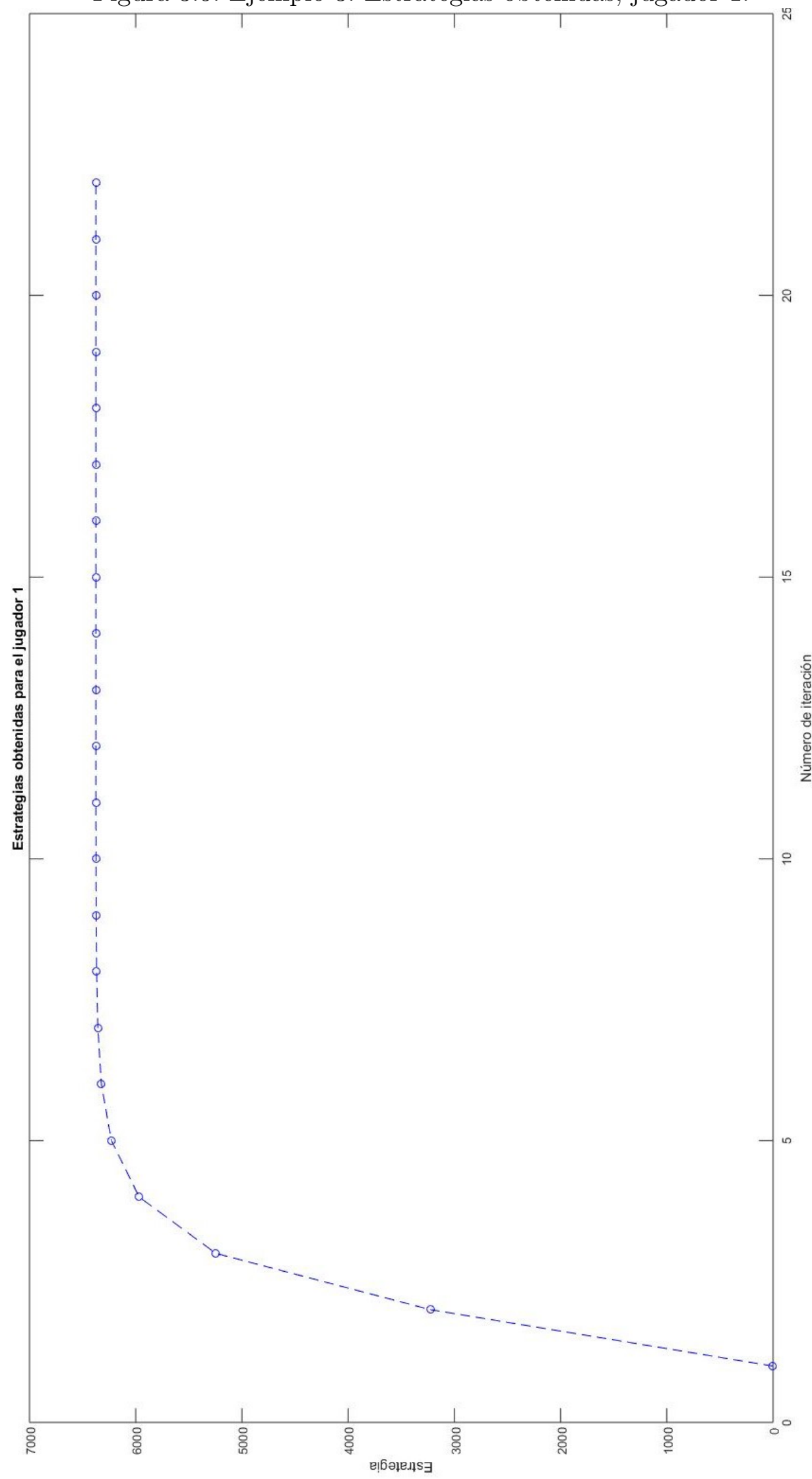


Figura 3.6: Ejemplo 3. Estrategias obtenidas, jugador 2.

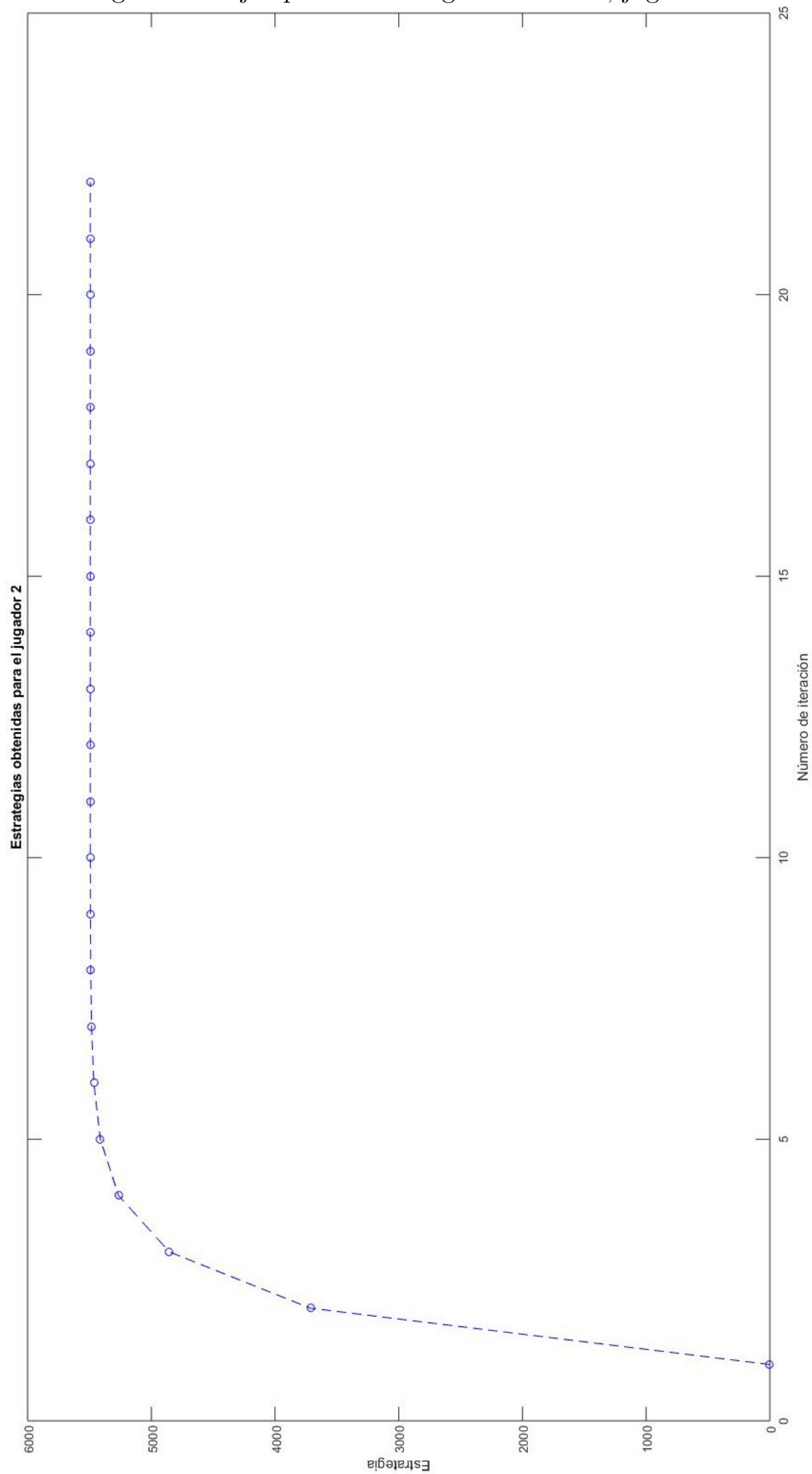




\subsubsection{Comentarios sobre demandas distribuidas exponen- cialmente}

En este apartado, se presenta la forma de las funciones de utilidad esperada cuando las demandas se distribuyen exponencialmente, seguido de esto se muestra que dichas funciones son supermodulares, siempre que los parámetros de las demandas cumplan ciertas condiciones.

\section{Utilidad con demandas distribuidas exponencialmente}

Considere dos jugadores, con demandas, $D_{0}, D_{1}$ y $D_{2}$ distribuidas exponencialmente, con parámetros $\lambda_{1}, \lambda_{2}$ y $\lambda_{3}$, respectivamente; tales que:

$$
\lambda_{0}-\lambda_{1}>0, \quad \lambda_{0}-\lambda_{2}>0 \quad \text { y } \quad \lambda_{0}-\lambda_{1}-\lambda_{2}>0
$$

La función de utilidad para el jugador $i$, dada en (B.1), queda como:

$$
\begin{aligned}
E \Pi_{i}\left(Q_{i} \mid Q_{k}\right)= & p_{i} Q_{i} P\left(Q_{i}<D_{i}\right)+p_{i} \int_{0}^{\infty} y \mathbb{1}_{\left[y \leq Q_{i}\right]} f_{i}(y) d y-c_{i} Q_{i} \\
& +p_{0 i} \int_{0}^{\infty}\left\{E\left[D_{0} \mathbb{1}_{\left[D_{0}<Q_{i}-D_{i}\right]} \mathbb{1}_{\left[D_{0}<Q_{k}-D_{k}\right]} \mid D_{0}=y\right] f_{0}(y)\right. \\
& +Q_{i} E\left[\mathbb{1}_{\left[D_{i}<Q_{i}\right]} \mathbb{1}_{\left[Q_{i}<D_{i}+D_{0}\right]} \mathbb{1}_{\left[Q_{i}<D_{i}+Q_{k}-D_{k}\right]} \mid D_{i}=y\right] f_{i}(y) \\
& -E\left[D_{i} \mathbb{1}_{\left[D_{i}<Q_{i}\right]} \mathbb{1}_{\left[Q_{i}<D_{i}+D_{0}\right]} \mathbb{1}_{\left[Q_{i}<D_{i}+Q_{k}-D_{k}\right]} \mid D_{i}=y\right] f_{i}(y) \\
& +Q_{k} E\left[\mathbb{1}_{\left[D_{k}<Q_{k}\right]} \mathbb{1}_{\left[Q_{k}<D_{k}+D_{0}\right]} \mathbb{1}_{\left[Q_{k}<D_{k}+Q_{i}-D_{i}\right]} \mid D_{k}=y\right] f_{k}(y) \\
& \left.-E\left[D_{k} \mathbb{1}_{\left[D_{k}<Q_{k}\right]} \mathbb{1}_{\left[Q_{k}<D_{k}+D_{0}\right]} \mathbb{1}_{\left[Q_{k}<D_{k}+Q_{i}-D_{i}\right]} \mid D_{k}=y\right] f_{k}(y)\right\} d y
\end{aligned}
$$

donde $\mathbb{1}_{A}$ denota la función indicadora del conjunto $A$. 
Por la independencia de las demandas, se tiene:

$$
\begin{aligned}
& =p_{i} Q_{i} P\left(Q_{i}<D_{i}\right)+p_{i} \int_{0}^{Q_{i}} y f_{i}(y) d y-c Q_{i} \\
& +p_{0 i} \int_{0}^{\infty} y P\left(D_{i}<Q_{i}-y\right) P\left(D_{k}<Q_{k}-y\right) \lambda_{0} e^{-\lambda_{0} y} d y \\
& +p_{0 i} Q_{i} \int_{0}^{Q_{i}} P\left(Q_{i}-y<D_{0}\right) P\left(D_{k}<y+Q_{k}-Q_{i}\right) \lambda_{i} e^{-\lambda_{i} y} d y \\
& -p_{0 i} \int_{0}^{Q_{i}} y P\left(D_{0}<Q_{i}-y\right) P\left(D_{k}<y+Q_{k}-Q_{i}\right) \lambda_{i} e^{-\lambda_{i} y} d y \\
& +p_{0 i} Q_{k} \int_{0}^{Q_{k}} P\left(Q_{k}-y<D_{0}\right) P\left(D_{i}<y+Q_{i}-Q_{k}\right) \lambda_{k} e^{-\lambda_{k} y} d y \\
& -p_{0 i} \int_{0}^{Q_{k}} y P\left(D_{0}<Q_{k}-y\right) P\left(D_{i}<y+Q_{i}-Q_{k}\right) \lambda_{k} e^{-\lambda_{k} y} d y
\end{aligned}
$$

Resolviendo las integrales, se obtiene

$$
\begin{aligned}
& =p_{i} Q_{i} e^{-\lambda_{i} Q_{i}}+p_{i} e^{-\lambda_{i} Q_{i}}\left(Q_{i}-\frac{1}{\lambda_{i}}\right)+\frac{p_{i}}{\lambda_{i}} \\
& +p_{0 i}\left\{\lambda_{0}\left(\frac{1}{\lambda_{0}^{2}}-\frac{e^{-\lambda_{i} Q_{i}}}{\left(\lambda_{0}-\lambda_{i}\right)^{2}}-\frac{e^{-\lambda_{k} Q_{k}}}{\left(\lambda_{0}-\lambda_{k}\right)^{2}}+\frac{e^{-\lambda_{i} Q_{i}-\lambda_{k} Q_{k}}}{\left(\lambda_{0}-\lambda_{i}-\lambda_{k}\right)^{2}}\right)\right. \\
& +\lambda_{i} Q_{i}\left[\frac{e^{-\lambda_{i} Q_{i}}-e^{-\lambda_{0} Q_{i}}}{\lambda_{0}-\lambda_{i}}-\left(\frac{e^{-\lambda_{k} Q_{k}-\lambda_{i} Q_{i}}-e^{-\lambda_{0} Q_{i}-\lambda_{k} Q_{k}+\lambda_{k} Q_{i}}}{\lambda_{0}-\lambda_{i}-\lambda_{k}}\right)\right] \\
& -\lambda_{i}\left[\frac{Q_{i} e^{-\lambda_{i} Q_{i}}}{\lambda_{0}-\lambda_{i}}-\left(\frac{e^{-\lambda_{i} Q_{i}}-e^{-\lambda_{0} Q_{i}}}{\left(\lambda_{0}-\lambda_{i}\right)^{2}}\right)-\frac{Q_{i} e^{-\lambda_{k} Q_{k}-\lambda_{i} Q_{i}}}{\lambda_{0}-\lambda_{i}-\lambda_{k}}\right. \\
& \left.+\left(\frac{e^{-\lambda_{i} Q_{i}-\lambda_{k} Q_{k}}-e^{-\lambda_{0} Q_{i}-\lambda_{k} Q_{k}+\lambda_{k} Q_{i}}}{\left(\lambda_{0}-\lambda_{i}-\lambda_{k}\right)^{2}}\right)\right] \\
& +\lambda_{k} Q_{k}\left[\frac{e^{-\lambda_{k} Q_{k}}-e^{-\lambda_{0} Q_{k}}}{\lambda_{0}-\lambda_{k}}-\left(\frac{e^{-\lambda_{i} Q_{i}-\lambda_{k} Q_{k}}-e^{-\lambda_{0} Q_{k}-\lambda_{i} Q_{i}+\lambda_{i} Q_{k}}}{\lambda_{0}-\lambda_{k}-\lambda_{i}}\right)\right] \\
& -\lambda_{k}\left[\frac{Q_{k} e^{-\lambda_{k} Q_{k}}}{\lambda_{0}-\lambda_{k}}-\left(\frac{e^{-\lambda_{k} Q_{k}}-e^{-\lambda_{0} Q_{k}}}{\left(\lambda_{0}-\lambda_{k}\right)^{2}}\right)-\frac{Q_{k} e^{-\lambda_{i} Q_{i}-\lambda_{k} Q_{k}}}{\lambda_{0}-\lambda_{k}-\lambda_{i}}\right. \\
& \left.\left.+\left(\frac{e^{-\lambda_{k} Q_{k}-\lambda_{i} Q_{i}}-e^{-\lambda_{0} Q_{k}-\lambda_{i} Q_{i}+\lambda_{i} Q_{k}}}{\left(\lambda_{0}-\lambda_{k}-\lambda_{i}\right)^{2}}\right)\right]\right\}-c_{i} Q_{i}
\end{aligned}
$$


Simplificando, la función de utilidad esperada, cuando las demandas son exponenciales, queda de la forma:

$$
\begin{aligned}
& =p_{i} e^{-\lambda_{i} Q_{i}}\left(2 Q_{i}-\frac{1}{\lambda_{i}}\right)+\frac{p_{i}}{\lambda_{i}}+\frac{p_{0 i}}{\lambda_{0}}+\frac{p_{0 i} e^{-\lambda_{i} Q_{i}}}{\lambda_{i}-\lambda_{0}} \\
& +\frac{p_{0 i} e^{-\lambda_{k} Q_{k}}}{\lambda_{k}-\lambda_{0}}+\frac{p_{0 i} e^{-\lambda_{i} Q_{i}-\lambda_{k} Q_{k}}}{\lambda_{0}-\lambda_{i}-\lambda_{k}}-\frac{p_{0 i} \lambda_{i} Q_{i} e^{-\lambda_{0} Q_{i}}}{\lambda_{0}-\lambda_{i}} \\
& +\frac{p_{0 i} \lambda_{i} Q_{i} e^{-\lambda_{0} Q_{i}-\lambda_{k} Q_{k}+\lambda_{k} Q_{i}}}{\lambda_{0}-\lambda_{i}-\lambda_{k}}-\frac{p_{0 i} \lambda_{i} e^{-\lambda_{0} Q_{i}}}{\left(\lambda_{i}-\lambda_{0}\right)^{2}} \\
& +\frac{p_{0 i} \lambda_{i} e^{-\lambda_{0} Q_{i}-\lambda_{k} Q_{k}+\lambda_{k} Q_{i}}}{\left(\lambda_{i}+\lambda_{k}-\lambda_{0}\right)^{2}}-\frac{p_{0 i} \lambda_{k} Q_{k} e^{-\lambda_{0} Q_{k}}}{\lambda_{0}-\lambda_{k}} \\
& +\frac{p_{0 i} \lambda_{k} Q_{k} e^{-\lambda_{i} Q_{i}+\lambda_{i} Q_{k}-\lambda_{0} Q_{k}}}{\lambda_{0}-\lambda_{k}-\lambda_{i}}-\frac{p_{0 i} \lambda_{k} e^{-\lambda_{0} Q_{k}}}{\left(\lambda_{k}-\lambda_{0}\right)^{2}} \\
& +\frac{p_{0 i} \lambda_{k} e^{-\lambda_{0} Q_{k}-\lambda_{i} Q_{i}+\lambda_{i} Q_{k}}}{\left(\lambda_{i}+\lambda_{k}-\lambda_{0}\right)^{2}}-c_{i} Q_{i}
\end{aligned}
$$

\section{Diferencias crecientes}

Se probará que la función de utilidad esperada, obtenida en (3.13), tiene diferencias crecientes, esto mostrando que su segunda derivada es no negativa y haciendo uso del teorema 1.48 .

La primera derivada de la función de utilidad esperada está dada por:

$$
\begin{aligned}
\frac{\partial}{\partial Q_{k}} E \Pi_{i}\left(Q_{i} \mid Q_{k}\right)= & \frac{p_{0 i} \lambda_{k} e^{\lambda_{k} Q_{k}}}{\lambda_{0}-\lambda_{k}}-\frac{p_{0 i} \lambda_{k} e^{-\lambda_{i} Q_{i}-\lambda_{k} Q_{k}}}{\lambda_{0}-\lambda_{i}-\lambda_{k}} \\
& -\frac{p_{0 i} \lambda_{i} \lambda_{k} Q_{i} e^{-\lambda_{0} Q_{i}+\lambda_{k} Q_{i}-\lambda_{k} Q_{k}}}{\lambda_{0}-\lambda_{i}-\lambda_{k}}+\frac{p_{0 i} \lambda_{i} \lambda_{k} Q_{i} e^{-\lambda_{0} Q_{i}+\lambda_{k} Q_{i}-\lambda_{k} Q_{k}}}{\left(\lambda_{0}-\lambda_{i}-\lambda_{k}\right)^{2}} \\
& -\frac{p_{0 i} \lambda_{k}}{\lambda_{0}-\lambda_{k}}\left(e^{-\lambda_{0} Q_{k}}-\lambda_{0} Q_{k} e^{-\lambda_{0} Q_{k}}\right) \\
& +\frac{p_{0 i} \lambda_{k} e^{-\lambda_{i} Q_{i}}}{\lambda_{0}-\lambda_{k}-\lambda_{i}}\left[e^{\lambda_{i} Q_{k}-\lambda_{0} Q_{k}}+Q_{k} e^{\lambda_{i} Q_{k}-\lambda_{0} Q_{k}}\left(\lambda_{i}-\lambda_{0}\right)\right] \\
& +\frac{p_{0 i} \lambda_{k} \lambda_{0} e^{-\lambda_{0} Q_{k}}}{\left(\lambda_{k}-\lambda_{0}\right)^{2}}+\frac{p_{0 i} \lambda_{k}\left(\lambda_{i}-\lambda_{0}\right) e^{-\lambda_{i} Q_{i}-\lambda_{0} Q_{k}+\lambda_{i} Q_{k}}}{\left(\lambda_{0}-\lambda_{i}-\lambda_{k}\right)^{2}}
\end{aligned}
$$


La segunda derivada de la función de utilidad, es:

$$
\begin{aligned}
\frac{\partial^{2}}{\partial Q_{i} \partial Q_{k}} E \Pi_{i}\left(Q_{i} \mid Q_{k}\right)= & \frac{p_{0 i} \lambda_{i} \lambda_{k} e^{-\lambda_{k} Q_{k}-\lambda_{i} Q_{i}}}{\lambda_{0}-\lambda_{k}-\lambda_{i}}-\frac{p_{0 i} \lambda_{i} \lambda_{k} e^{-\lambda_{k} Q_{k}}}{\lambda_{0}-\lambda_{i}-\lambda_{k}}\left[e^{-\lambda_{0} Q_{i}+\lambda_{k} Q_{i}}\right. \\
& \left.+\left(\lambda_{k}-\lambda_{0}\right) Q_{i} e^{-\lambda_{0} Q_{i}+\lambda_{k} Q_{i}}\right] \\
& -\frac{p_{0 i} \lambda_{i} \lambda_{k} e^{-\lambda_{k} Q_{k}-\lambda_{0} Q_{i}+\lambda_{k} Q_{i}}\left(\lambda_{k}-\lambda_{0}\right)}{\left(\lambda_{i}+\lambda_{k}-\lambda_{0}\right)^{2}} \\
& -\frac{p_{0 i} \lambda_{i} \lambda_{k} e^{-\lambda_{i} Q_{i}}}{\lambda_{0}-\lambda_{i}-\lambda_{k}}\left[e^{-\lambda_{0} Q_{k}+\lambda_{i} Q_{k}}+\left(\lambda_{i}-\lambda_{0}\right) Q_{k} e^{-\lambda_{0} Q_{k}+\lambda_{i} Q_{k}}\right] \\
& -\frac{p_{0 i} \lambda_{i} \lambda_{k} e^{-\lambda_{i} Q_{i}-\lambda_{0} Q_{k}+\lambda_{i} Q_{k}}\left(\lambda_{i}-\lambda_{0}\right)}{\left(\lambda_{i}+\lambda_{k}-\lambda_{0}\right)^{2}}
\end{aligned}
$$

Simplificando, se tiene:

$$
\begin{aligned}
& =\frac{p_{0 i} \lambda_{i} \lambda_{k} e^{-\lambda_{k} Q_{k}-\lambda_{i} Q_{i}}}{\lambda_{0}-\lambda_{k}-\lambda_{i}} \\
& +\left(\frac{\lambda_{0}-\lambda_{k}}{\lambda_{0}-\lambda_{i}-\lambda_{k}}-1\right) \frac{p_{0 i} \lambda_{k} \lambda_{i} e^{-\lambda_{k} Q_{k}-\lambda_{0} Q_{i}+\lambda_{k} Q_{i}}}{\left(\lambda_{0}-\lambda_{i}-\lambda_{k}\right)^{2}} \\
& +\frac{\left(\lambda_{0}-\lambda_{k}\right) p_{0 i} \lambda_{i} \lambda_{k} Q_{i} e^{-\lambda_{k} Q_{k}-\lambda_{0} Q_{i}+\lambda_{k} Q_{i}}}{\lambda_{0}-\lambda_{i}-\lambda_{k}} \\
& +\left(\frac{\lambda_{0}-\lambda_{i}}{\lambda_{0}-\lambda_{i}-\lambda_{k}}-1\right) \frac{p_{0 i} \lambda_{k} \lambda_{i} e^{-\lambda_{i} Q_{i}-\lambda_{0} Q_{k}+\lambda_{i} Q_{k}}}{\left(\lambda_{0}-\lambda_{i}-\lambda_{k}\right)^{2}} \\
& +\frac{\left(\lambda_{0}-\lambda_{i}\right) p_{0 i} \lambda_{i} \lambda_{k} Q_{k} e^{-\lambda_{i} Q_{i}-\lambda_{0} Q_{k}+\lambda_{i} Q_{k}}}{\lambda_{0}-\lambda_{i}-\lambda_{k}}
\end{aligned}
$$

Por las condiciones (3.12), se tiene que la segunda derivada de la función de utilidad esperada, (3.15), es no negativa, de esto que tenga diferencias crecientes y por ende sea supermodular.

Así, el modelo de productos parcialmente complementarios con demandas exponenciales, también se puede ver como un juego supermodular no cooperativo cuando los parámetros de las exponenciales cumplen las condiciones (3.12). 


\subsection{Conclusiones}

En este capítulo, se presentaron algunos modelos en los cuales se han utilizado los juegos supermodulares no cooperativos, así mismo, se planteó un modelo que en la bibliografía se había presentado como un juego no cooperativo y se ejemplifica como, al dar más especificaciones sobre las demandas, es posible abordar dicho modelo como un juego supermodular y aproximar equilibrios de manera numérica usando los algoritmos planteados en el capítulo 2. 


\section{Capítulo 4}

\section{Conclusiones y perspectivas}

En esta tesis, se presentaron los conceptos básicos, necesarios para adentrarse en el estudio de los juegos supermodulares no cooperativos, se plantearon ideas importantes de estos y se muestran algunas aplicaciones de dichos juegos, así como sus soluciones teóricas o numéricas, mejor conocidas como equilibrios de Nash.

La tesis se enfocó en los juegos no cooperativos, en estos, los jugadores toman decisiones independientemente unos de otros.

Se consideró importante el estudio de los juegos supermodulares no cooperativos pues en estos la existencia de puntos de equilibrio se asegura sin hacer uso de teoremas de punto fijo y, en la literatura especializada en el tema se pueden encontrar algoritmos para aproximar dichos equilibrios. Así, en este tipo de juegos no solo se garantiza la existencia de equilibrios, también es posible aproximarlos.

Se encontró que los juegos supermodulares no cooperativos son ampliamente usados en economía, investigación de operaciones, etc.; para ver algunos ejemplos de estas aplicaciones, ver capítulo 3.

Por otra parte, en esta tesis se presentó una aplicación con un ambiente estocástico, situación que hasta el momento no se encontró en la literatura pues, en las aplicaciones ya conocidas, las funciones de utilidad son deterministas y aquí se plantea un modelo aleatorio, basado en el juego no cooperativo de productos parcialmente complementarios descrito en el artículo Capacity Games for Partially Complementary Products Under Multivariate Random Demands, de Xiang Fang [2]. 
En el trabajo a futuro se considera:

- Estudiar la posibilidad de ampliar la cota de iteraciones en los algoritmos de aproximación de equilibrios a conjuntos compactos no necesariamente finitos.

En el modelo de productos parcialmente complementarios:

- estudiar el caso en el que los precios de venta en conjunto son mayores que los de venta individual y el caso en el que la relación entre los precios, de venta individual y conjunta, varíe dependiendo del jugador,

- investigar para qué otras distribuciones de las demandas las funciones de utilidad son supermodulares,

- analizar el modelo cuando las demandas no son independientes. 


\section{Apéndice A}

\section{Algunas demostraciones}

\section{Demostración (Lema 1.20)}

La prueba se realizará usando inducción.

Considere $X$ una latiz, $Y$ una sublatiz de esta y $Z=\left\{x_{1}, \ldots, x_{k}\right\} \subset Y$, con $k \in \mathbb{N}$.

Defínanse $y_{1}=x_{1} \in Y$ y $y_{2}=\sup _{X}\left\{x_{1}, x_{2}\right\}=x_{1} \vee x_{2} \in Y$.

Hipótesis inductiva: Para $1 \leq n<k, y_{n}=\sup _{X}\left\{x_{1}, \ldots, x_{n}\right\} \in Y$.

Sea $y_{n+1}=y_{n} \vee x_{n+1}$, como $Y$ es una latiz, $y_{n+1} \in Y$. Note que $y_{n+1}$ es una cota superior del conjunto $\left\{x_{1}, \ldots, x_{n+1}\right\}$, se probará que esta es la mínima.

Sea $y \in Y$ otra cota superior del conjunto $\left\{x_{1}, \ldots, x_{n+1}\right\}$, como $y_{n}$ es el supremo de $\left\{x_{1}, \ldots, x_{n}\right\}$, se tiene que $y_{n} \preceq y$, además $x_{n+1} \preceq y$, de esto que $y_{n+1}=x_{n+1} \vee y_{n} \preceq y$, así $y_{n+1}=\sup _{X}\left\{x_{1}, \ldots, x_{n+1}\right\}$.

Luego, para $n=k-1$, se tiene que $y_{k}=\sup _{X}(Z)$ y $y_{k} \in Y$.

De manera análoga, sea $y_{1}=x_{1} \in Y, y_{2}=\inf _{X}\left\{x_{1}, x_{2}\right\}=x_{1} \wedge x_{2} \in Y$.

Ahora, suponga que, para $1 \leq n<k, y_{n}=\inf _{X}\left\{x_{1}, \ldots, x_{n}\right\} \in Y$. Considere $y_{n+1}=y_{n} \wedge x_{n+1}, y_{n+1} \in Y$, pues $Y$ es una latiz; además, $y_{n+1}$ es una cota inferior del conjunto $\left\{x_{1}, \ldots, x_{n+1}\right\}$.

Sea $y \in Y$ otra cota inferior de $\left\{x_{1}, \ldots, x_{n+1}\right\}$, así $y \preceq y_{n}$ y $y \preceq x_{n+1}$, luego, $y \preceq x_{n+1} \wedge y_{n}=y_{n+1}$, se sigue que $y_{n+1}=\inf _{X}\{Z\}$.

Finalmente, si $X$ es una latiz finita, considere $Z=Y=Z$ y se concluye que $X$ tiene supremo e ínfimo. 
Demostración (Teorema 1.31)

$\Rightarrow$ ] Considere $X \subset \mathbb{R}^{n}$ una sublatiz subcompleta, $X \neq \emptyset$. Se probará que $X$ es un subconjunto cerrado y acotado de $\mathbb{R}^{n}$.

Como $X$ es una sublatiz subcompleta, tiene elementos máximo y mínimo, de esto que $X$ sea un conjunto acotado.

Ahora, considere $x \in \operatorname{cl}(X)$, entonces existe $\left\{x_{n}\right\}_{n \in \mathbb{N}} \subset X$, tal que $x_{n} \rightarrow x$. Por la subcompletez de $X$, existen $z^{k}=\sup _{\mathbb{R}^{n}}\left\{x_{n}: n=k, k+1, \ldots\right\}, \forall k \in \mathbb{N}$ y $x^{*}=\inf _{\mathbb{R}^{n}}\left\{z^{k}: k \in \mathbb{N}\right\}$ y son tales que $x^{*}, z^{k} \in X, \forall k \in \mathbb{N}$.

Note que, $z^{1} \geq z^{2} \geq \ldots$. Así

$$
x=\lim _{n \rightarrow \infty} x_{n}=\lim _{k \rightarrow \infty} z^{k}=x^{*} \in X
$$

esto pues $x_{n} \rightarrow x$ y los $z^{k}$ son una subsucesión de $x_{n}$, así $z^{k} \rightarrow x$, además $\left\{z^{k}\right\}_{k \in \mathbb{N}}$ es una subsucesión decreciente acotada inferiormente por $x^{*}$, de esto que se de la tercera igualdad en (A.1).

Así $X$ es cerrado y se concluye, por el teorema de Heine-Borel, que $X$ es un conjunto compacto.

$\Leftarrow]$ Se probará que, para cualquier subconjunto no vacío de la sublatiz compacta, sus elementos supremo e ínfimo están en ella.

Sea $X$ una sublatiz compacta de $\mathbb{R}^{n}$ y $Y \subset X, Y \neq \emptyset$. Como $X$ es compacto, $c l(Y)$ también lo es.

Ahora, para $i=1, \ldots, n$, considere

$$
\operatorname{argmín}_{x \in c l(Y)} x_{i}=\left\{x \in \operatorname{cl}(Y): x_{i} \leq y_{i}, \forall y \in \operatorname{cl}(Y)\right\}
$$

Para todo $i \in\{1, \ldots, n\}$, tómese $x^{i} \in \operatorname{argmín}_{x \in c l(Y)} x_{i}$. Luego, se puede definir $x^{*}=\inf _{\mathbb{R}^{n}}\left\{x^{1}, \ldots, x^{n}\right\}=\left(x_{1}{ }^{1} \ldots, x_{n}{ }^{n}\right)$, donde $x_{i}{ }^{i}$ representa la $i$-ésima entrada del $x^{i}$ elegido anteriomente. Del lema 1.20, se tiene que $x^{*} \in X$.

Observe que, para $x \in c l(Y), x_{i} \geq x_{i}{ }^{i}=x_{i}{ }^{*}$, con $i=1, \ldots, n$, de esto que $x^{*}$ sea una cota inferior para $c l(Y)$, por ende, también sea una cota inferior para $Y$.

Sea $z$ otra cota inferior para $Y$, este elemento también es una cota inferior para $c l(Y)$ y es tal que $x^{i} \geq z, \forall i \in\{1, \ldots, n\}$, así $x^{*} \geq z$, de aquí que $x^{*}=\inf _{\mathbb{R}^{n}}\{Y\}$. 
De manera análoga, se puede probar que $w^{*}=\sup _{\mathbb{R}^{n}}\{Y\}$ pertenece a $X$, de esto que $X$ sea una sublatiz subcompleta.

\section{Demostración (Teorema 1.41)}

a) Inicialmente se probará que el conjunto de puntos fijos de la correspondencia Y es no vacío.

Defínase $B=\{x \in X: Y(x) \cap(-\infty, x] \neq \emptyset\}$. Como $X$ es una latiz completa, se tiene que $\sup _{X}\{X\}$ existe; luego, $Y\left(\sup _{X}\{X\}\right) \neq \emptyset$, pues es un elemento de $\mathscr{L}(X)$, además, $Y\left(\sup _{X}\{X\}\right) \cap\left(-\infty, \sup _{X}\{X\}\right] \neq \emptyset$, de aquí que $B \neq \emptyset$ y, dado que $X$ es una latiz completa, existe $b=\inf _{x}\{B\}$.

Considere $y=\inf _{X}\{Y(b)\}$, este elemento existe pues, $Y(b)$ es una sublatiz subcompleta. Ahora, como $b \in B$, existe $z \in Y(b)$ tal que $y \preceq z \preceq b$, luego $y \preceq b$ y la correspondencia Y es creciente, así $Y(y) \sqsubseteq Y(b)$.

Sea $\beta=\inf _{X}\{Y(y)\}$, por el lema 1.35, se tiene que $\beta \preceq y$, así $y \in B$ y $y \preceq b \preceq y$, entonces $y=b=\inf _{X}\{B\}$, luego $b \in Y(b)$, de aquí que $b$ sea un punto fijo de $Y(b)$. Así, el conjunto de puntos fijos de la correspondencia $Y$ es no vacío.

Como los puntos fijos de la correspondencia Y están en $B$, se tiene que $b=$ $\inf _{X}\{B\}$ es el mínimo punto fijo de $Y$.

La prueba de que $\sup _{X}\{x \in X: Y(x) \cap[x, \infty) \neq \emptyset\}$ es el máximo punto fijo de Y es análoga.

b) Del inciso anterior, se tiene que el conjunto de puntos fijos de $\mathrm{Y}$ es no vacío. Ahora, se probará que dicho conjunto es una latiz completa.

Sea $E$ el conjunto de puntos fijos de Y y $U \subset E$. Considere $b=\sup _{X}\{U\}$. Para cualquier $e \in U$, se tiene que $e \in Y(e)$, pues $e$ es un punto fijo.

Ahora, $\mathrm{Y}$ es creciente, así $e \preceq b$, luego, existe $x_{e} \in Y(b)$ tal que $e \preceq x_{e}$. Sea $x=\sup _{X}\left\{x_{e}: e \in U\right\}$, se tiene que $e \preceq x_{e}$, así $b \preceq x$. además $x \in Y(b)$, pues $Y(b)$ es una sublatiz subcompleta.

Luego, sea $S^{\prime}=\left[b, \sup _{X}\{X\}\right]$ y, definimos $g(s)=Y(s) \cap\left[b, \sup _{X}\{X\}\right], \forall s \in S^{\prime}$. Se tiene que $g(s)$ es no vacío para cada $s \in S^{\prime}$, además, $Y(s)$ y $\left[b, \sup _{X}\{X\}\right]$ son 
sublatices subcompletas, así $g(s)$ es una sublatiz subcompleta, para todo $s \in S^{\prime}$. Defínase $h(s)=\left[b, \sup _{X}\{X\}\right], \forall s \in S^{\prime}$. Las correspondencias $h$ y Y son crecientes, entonces $g=h \cap \mathrm{Y}$ es creciente en $S^{\prime}$, luego $g$ cumple las hipótesis del teorema y el conjunto de puntos fijos de $g$ es no vacío y tiene elementos máximo y mínimo.

Sea $b^{\prime}$ el ínfimo de los puntos fijos de $g, b^{\prime}$ está en el conjunto de puntos fijos de $g$, denote por $E^{\prime}$ dicho conjunto, observe que $E^{\prime}=E \cap\left[b, \sup _{X}\{X\}\right]$, entonces $b^{\prime}$ es el mínimo punto fijo mayor o igual que $b$, de esto que $b^{\prime}=\sup _{E} U$.

De manera análoga, se muestra que existe $a^{\prime}=\inf _{E} U$, así, cualquier subconjunto de $E$ tiene supremo e ínfimo en $E$, de aquí que $E$ sea una latiz completa.

\section{Demostración (Lema 2.11)}

a) Los conjuntos $S_{i}\left(x_{-i}\right)$ son secciones de una sublatiz, de esto que, por el teorema 1.38, sean conjuntos crecientes. Ahora, las funciones de pago son supermodulares y tienen diferencias crecientes, así, del teorema 1.64, se sigue que la correspondencia de mejor respuesta para cada jugador, $Y_{i}\left(x_{-i}\right)$, es creciente en $x_{i} \in S_{-i}$.

De lo anterior y el teorema 1.66, se tiene que el elemento máximo (mínimo) de $Y_{i}\left(x_{-i}\right)$ es creciente en $S_{i}$.

Se procederá a probar que el conjunto de mejores respuestas del jugador $i$ es una sublatiz compacta y no vacía de $\mathbb{R}^{m_{i}}$, esto se obtendrá por el corolario 1.61, basta probar que $S_{i}\left(x_{-i}\right)$ es una sublatiz compacta de $\mathbb{R}^{m_{i}}$.

Para ello, se probará que es un conjunto cerrado, teniendo esto, es fácil ver que $S_{i}\left(x_{-i}\right) \times\left\{x_{-i}\right\}$ es un subconjunto cerrado de un compacto, de esto que sea compacto y por ello, se concluirá que $S_{i}\left(x_{-i}\right)$ es compacto.

Recuerde que $S_{i}\left(x_{-i}\right)$ es la sección de $S$ en $x_{-i}$, así $S_{i}\left(x_{-i}\right)$ es una sublatiz de $\mathbb{R}^{m_{i}}$, esto por el lema 1.23 .

Para ver que es cerrada, observe que el conjunto contiene a sus puntos límite pues, sea $a_{i}$ un punto límite de $S_{i}\left(x_{-i}\right)$ entonces, existe $\left\{x_{i}\right\}_{i \in \mathbb{N}} \subset S_{i}\left(x_{-i}\right)$ tal que, $x_{i} \rightarrow a_{i}$. Note que $\left(x_{-i} ; x_{i}\right) \in S, \forall i \in \mathbb{N}$, además, $\left(x_{-i} ; x_{i}\right) \rightarrow\left(x_{-i} ; a_{i}\right)$, así, dado que $S$ es cerrado, se tiene que $\left(x_{-i} ; a_{i}\right) \in S$. Luego, $a_{i} \in S_{i}\left(x_{-i}\right)$, de aquí que $S_{i}\left(x_{-i}\right)$ sea cerrado y por el argumento dado anteriormente, sea compacto. Así $Y_{i}\left(x_{-i}\right)$ es una sublatiz subcompleta y no vacía de $\mathbb{R}^{n}$.

b) Se sabe que $S_{i}\left(x_{-i}\right)$ son conjuntos crecientes, así $\prod_{i \in N} S_{i}\left(x_{-i}\right)$ es creciente, luego $S(\mathbf{x})=\prod_{i \in N} S_{i}\left(x_{-i}\right) \cap S$ es creciente, las funciones de utilidad, $R_{i}$, tienen 
diferencias crecientes, de aquí que $g$ también tenga diferencias crecientes y sea supermodular. Luego, por el teorema 1.64, se tiene que $Y(\mathbf{x})$ es creciente para $\mathbf{x} \in S$. Ahora, por el teorema 1.66, se tiene que los elementos máximo y mínimo de la correspondencia de mejor respuesta conjunta son crecientes en $S$.

Falta ver que $Y(\mathbf{x})$ es una sublatiz compacta y no vacía de $\mathbb{R}^{m}$. Para ello, recuerde que $S(\mathbf{x})=S \cap \prod_{i}^{n} S_{i}\left(x_{-i}\right) S$, ambos intersectandos son sublatices compactas, así, $S(\mathbf{x})$ es una sublatiz compacta, para cada $\mathbf{x} \in S$. Luego, como $R_{i}$ es semicontinua superiormente en $x_{i} \in S_{i}\left(x_{-i}\right), g$ también lo es, en $S(\mathbf{x})$. De manera similar, como $R_{i}$ es supermodular en $y_{i} \in S_{i}$, se tiene que $g$ es supermodular en $\mathbf{y} \in S(\mathbf{x})$. Con esto se puede usar el corolario 1.61 y concluir que $Y(\mathbf{x})$ es una sublatiz compacta y no vacía de $\mathbb{R}^{m}$. 


\section{Apéndice B}

\section{Función de utilidad esperada}

La función de utilidad esperada, para el jugador $i$, en el modelo de productos parcialmente complementarios, se puede expresar de la siguiente manera:

$$
E \Pi_{i}\left(Q_{i} \mid Q_{k}\right)=E\left[p_{i} \operatorname{mín}\left\{D_{i}, Q_{i}\right\}+p_{0 i} \operatorname{mín}_{k=1 \ldots, n}\left\{D_{0},\left(Q_{k}-D_{k}\right)^{+}\right\}-c_{i} Q_{i}\right]
$$

separando la esperanza de la suma, se tiene

$$
\begin{aligned}
& =p_{i} E\left[D_{i} \mathbb{1}_{\left[D_{i} \leq Q_{i}\right]}\right]+p_{i} Q_{i} E\left[\mathbb{1}_{\left[Q_{i}<D_{i}\right]}\right]+p_{0 i}\left\{E\left[D_{0} \mathbb{1}_{\left[D_{0}<\min _{\forall k}\left(Q_{k}-D_{k}\right)^{+}\right]}\right]\right. \\
& \left.+\sum_{k=1}^{n} E\left[\left(Q_{k}-D_{k}\right)^{+} \mathbb{1}_{\left[\left(Q_{k}-D_{k}\right)^{+}<\min _{\forall j \neq k}\left\{D_{0},\left(Q_{j}-D_{j}\right)^{+}\right\}\right]}\right]\right\}-c_{i} Q_{i} \\
& =p_{i} E\left[D_{i} \mathbb{1}_{\left[D_{i} \leq Q_{i}\right]}\right]+p_{i} Q_{i} E\left[\mathbb{1}_{\left[Q_{i}<D_{i}\right]}\right]+p_{0 i} E\left[D_{0} \mathbb{1}_{\left[D_{0}<\operatorname{mí}_{\forall k}\left(Q_{k}-D_{k}\right)^{+}\right]}\right] \\
& +p_{0 i} \sum_{k=1}^{n}\left\{Q_{k} E\left[\mathbb{1}_{\left[D_{k}<Q_{k}<D_{k}+\min _{\forall j \neq k}\left\{D_{0},\left(Q_{j}-D_{j}\right)^{+}\right\}\right]}\right]\right. \\
& \left.-E\left[D_{k} \mathbb{1}_{\left[D_{k}<Q_{k}<D_{k}+\operatorname{mín}_{\forall j \neq k}\left\{D_{0},\left(Q_{j}-D_{j}\right)^{+}\right\}\right]}\right]\right\}-c_{i} Q_{i}
\end{aligned}
$$


usando propiedades de funciones indicadoras, la igualdad anterior se puede reescribir como

$$
\begin{aligned}
= & p_{i} E\left[D_{i} \mathbb{1}_{\left[D_{i} \leq Q_{i}\right]}\right]+p_{i} Q_{i} P\left(Q_{i}<D_{i}\right)+p_{0 i} E\left[D_{0} \mathbb{1}_{\left[D_{0}<\operatorname{mín}_{\forall k}\left(Q_{k}-D_{k}\right)^{+}\right]}\right] \\
& +p_{0 i} \sum_{k=1}^{n}\left\{Q_{k} P\left(D_{k}<Q_{k}<D_{k}+\min _{\forall j \neq k}\left\{D_{0},\left(Q_{j}-D_{j}\right)^{+}\right\}\right)\right. \\
& \left.-E\left[D_{k} \mathbb{1}_{\left[D_{k}<Q_{k}<D_{k}+\min _{\forall j \neq k}\left\{D_{0},\left(Q_{j}-D_{j}\right)^{+}\right\}\right]}\right]\right\}-c_{i} Q_{i}
\end{aligned}
$$

Ahora, considerando únicamente dos jugadores, la función de utilidad esperada, dada en (B.1), queda de la siguiente manera:

$$
\begin{aligned}
E \Pi_{i}\left(Q_{i} \mid Q_{k}\right)= & p_{i} Q_{i} P\left(Q_{i}<D_{i}\right)+p_{i} E\left[D_{i} \mathbb{1}_{\left[D_{i} \leq Q_{i}\right]}\right] \\
& +p_{0 i}\left\{E\left[D_{0} \mathbb{1}_{\left[D_{0}<\min _{j=1,2}\left(Q_{j}-D_{j}\right)^{+}\right]}\right]\right. \\
& +Q_{i} P\left(D_{i}<Q_{i}<D_{i}+\operatorname{mín}\left\{D_{0},\left(Q_{k}-D_{k}\right)^{+}\right\}\right) \\
& -E\left[D_{i} \mathbb{1}_{\left[D_{i}<Q_{i}<D_{i}+\min \left\{D_{0},\left(Q_{k}-D_{k}\right)^{+}\right\}\right]}\right] \\
& +Q_{k} P\left(D_{k}<Q_{k}<D_{k}+\operatorname{mín}\left\{D_{0},\left(Q_{i}-D_{i}\right)^{+}\right\}\right) \\
& -E\left[D_{k} \mathbb{1}_{\left[D_{k}<Q_{k}<D_{k}+\min \left\{D_{0},\left(Q_{i}-D_{i}\right)^{+}\right\}\right]}\right\}-c_{i} Q_{i}
\end{aligned}
$$

Nota B.1. Se considera que las demandas son independientes.

\section{B.1. Demandas distribuidas uniformemente}

Considere dos jugadores cuyas demandas están distribuidas uniformemente en el intervalo $[a, b]$. Usando propiedades de esperanza condicional [8], la función de 
utilidad esperada, (B.2), es de la forma:

$$
\begin{aligned}
E \Pi_{i}\left(Q_{i} \mid Q_{k}\right)= & p_{i} Q_{i} P\left(Q_{i}<D_{i}\right)+p_{i} \int_{a}^{b} y \mathbb{1}_{\left[y \leq Q_{i}\right]} f_{i}(y) d y-c_{i} Q_{i} \\
& +p_{0 i} \int_{a}^{b}\left\{E\left[D_{0} \mathbb{1}_{\left[D_{0}<Q_{i}-D_{i}\right]} \mathbb{1}_{\left[D_{0}<Q_{k}-D_{k}\right]} \mid D_{0}=y\right] f_{0}(y)\right. \\
& +Q_{i} E\left[\mathbb{1}_{\left[D_{i}<Q_{i}\right]} \mathbb{1}_{\left[Q_{i}<D_{i}+D_{0}\right]} \mathbb{1}_{\left[Q_{i}<D_{i}+Q_{k}-D_{k}\right]} \mid D_{i}=y\right] f_{i}(y) \\
& -E\left[D_{i} \mathbb{1}_{\left[D_{i}<Q_{i}\right]} \mathbb{1}_{\left[Q_{i}<D_{i}+D_{0}\right]} \mathbb{1}_{\left[Q_{i}<D_{i}+Q_{k}-D_{k}\right]} \mid D_{i}=y\right] f_{i}(y) \\
& +Q_{k} E\left[\mathbb{1}_{\left[D_{k}<Q_{k}\right]} \mathbb{1}_{\left[Q_{k}<D_{k}+D_{0}\right]} \mathbb{1}_{\left[Q_{k}<D_{k}+Q_{i}-D_{i}\right]} \mid D_{k}=y\right] f_{k}(y) \\
& \left.-E\left[D_{k} \mathbb{1}_{\left[D_{k}<Q_{k}\right]} \mathbb{1}_{\left[Q_{k}<D_{k}+D_{0}\right]} \mathbb{1}_{\left[Q_{k}<D_{k}+Q_{i}-D_{i}\right]} \mid D_{k}=y\right] f_{k}(y)\right\} d y
\end{aligned}
$$

sustituyendo la variable condicionante, es posible separar las esperanzas de las funciones indicadoras, obteniendo

$$
\begin{aligned}
= & p_{i} Q_{i} P\left(Q_{i}<D_{i}\right)+\frac{p_{i}}{b-a} \int_{a}^{\alpha_{i}} y d y-c_{i} Q_{i} \\
& +\frac{p_{0 i}}{b-a}\left\{\int_{a}^{b} y P\left(D_{i}<Q_{i}-y\right) P\left(D_{k}<Q_{k}-y\right) d y\right. \\
& +Q_{i} \int_{a}^{\alpha_{i}} P\left(D_{0}>Q_{i}-y\right) P\left(D_{k}<Q_{k}+y-Q_{i}\right) d y \\
& -\int_{a}^{\alpha_{i}} y P\left(D_{0}>Q_{i}-y\right) P\left(D_{k}<Q_{k}+y-Q_{i}\right) d y \\
& +Q_{k} \int_{a}^{\alpha_{k}} P\left(D_{0}>Q_{k}-y\right) P\left(D_{i}<Q_{i}+y-Q_{k}\right) d y \\
& \left.-\int_{a}^{\alpha_{k}} y P\left(D_{0}>Q_{k}-y\right) P\left(D_{i}<Q_{i}+y-Q_{k}\right) d y\right\}
\end{aligned}
$$

donde $\alpha_{\ell}=\min \left\{b, Q_{\ell}\right\}$, con $\ell=1,2$.

Dependiendo de los valores que tome $\alpha_{\ell}, \ell=1,2$, se tienen cuatro casos posibles en la igualdad (B.3). 
A continuación, se considera el caso en que $\alpha_{i}=Q_{i}$ y $\alpha_{k}=Q_{k}$.

$$
\begin{aligned}
E \Pi_{i}\left(Q_{i} \mid Q_{-i}\right)= & p_{i} Q_{i}\left(\frac{b-Q_{i}}{b-a}\right)+p_{i}\left(\frac{Q_{i}{ }^{2}-a^{2}}{2(b-a)}\right)+\frac{p_{0 i}}{(b-a)^{3}}\left[\frac{b^{4}-a^{4}}{4}\right. \\
& \left.+\left(2 a-Q_{k}-Q_{i}\right)\left(\frac{b^{3}-a^{3}}{3}\right)+\left(Q_{i} Q_{k}-a Q_{i}-a Q_{k}+a^{2}\right)\left(\frac{b^{2}-a^{2}}{2}\right)\right] \\
& +\sum_{\substack{j, s=1 \\
s \neq j}}^{2}\left\{\frac { p _ { 0 j } Q _ { j } } { ( b - a ) ^ { 3 } } \left[\frac{Q_{j}{ }^{3}-a^{3}}{3}+\left(b-2 Q_{j}+Q_{s}-a\right)\left(\frac{Q_{j}{ }^{2}-a^{2}}{2}\right)\right.\right. \\
& \left.+\left(Q_{j}-a\right)\left(b Q_{s}-b Q_{j}-b a-Q_{j} Q_{s}+Q_{j}{ }^{2}+a Q_{j}\right)\right] \\
& -\frac{p_{0 j}}{(b-a)^{3}}\left[\frac{Q_{j}{ }^{4}-a^{4}}{4}+\left(b-2 Q_{j}+Q_{s}-a\right)\left(\frac{Q_{j}{ }^{3}-a^{3}}{3}\right)\right. \\
& \left.\left.+\left(b Q_{s}-b Q_{j}-b a-Q_{j} Q_{s}+Q_{s}{ }^{2}+a Q_{j}\right)\left(\frac{Q_{j}{ }^{2}-a^{2}}{2}\right)\right]\right\}-c_{i} Q_{i}
\end{aligned}
$$

\section{B.1.1. Diferencias crecientes}

Considere la función de utilidad esperada dada en (B.3). Dicha función cumple las hipótesis del teorema 1.48, así para probar que la función de utilidad tiene diferencias crecientes basta ver que su segunda derivada es no negativa.

La primera derivada de la función de utilidad está dada por:

$$
\begin{aligned}
\frac{\partial}{\partial Q_{k}} E \Pi_{i}\left(Q_{i} \mid Q_{k}\right)= & \frac{p_{0 i}}{(b-a)^{3}} \int_{a}^{b}\left(Q_{i} y-y^{2}-a y\right)+\frac{p_{0 i} Q_{i}}{(b-a)^{3}} \int_{a}^{\alpha_{i}}\left(b+y-Q_{i}\right) d y \\
& -\frac{p_{0 i}}{(b-a)^{3}} \int_{a}^{\alpha_{i}}\left(b y-Q_{i}+y^{2}\right) d y \\
& +\frac{p_{0 i}}{(b-a)^{3}} \int_{a}^{\alpha_{k}}\left(b y+b Q_{i}-2 b Q_{k}-b a+y^{2}+Q_{i} y\right. \\
& \left.-4 Q_{k} y-a y-2 Q_{i} Q_{k}+3 Q_{k}^{2}+2 Q_{k} a\right) d y \\
& -\frac{p_{0 i}}{(b-a)^{3}} \int_{a}^{\alpha_{k}}\left(-b y-Q_{i} y-2 y^{2}+2 Q_{k} y+a y\right) d y
\end{aligned}
$$


La segunda derivada, queda de la forma:

$$
\begin{aligned}
\frac{\partial^{2}}{\partial Q_{i} \partial Q_{k}} E \Pi_{i}\left(Q_{i} \mid Q_{k}\right)= & \frac{p_{0 i}}{(b-a)^{3}} \int_{a}^{b} y d y+\frac{p_{0 i}}{(b-a)^{3}} \int_{a}^{\alpha_{i}}\left(b+y-2 Q_{i}\right) d y \\
& -\frac{p_{0 i}}{(b-a)^{3}} \int_{a}^{\alpha_{i}}(-y) d y+\frac{p_{0 i}}{(b-a)^{3}} \int_{a}^{\alpha_{k}}\left(b+y-2 Q_{k}\right) d y \\
& -\frac{p_{0 i}}{(b-a)^{3}} \int_{a}^{\alpha_{k}}(-y) d y \\
= & \frac{p_{0 i}}{(b-a)^{3}}\left[\frac{b^{2}-a^{2}}{2}+b \alpha_{i}-b a+\frac{\alpha_{i}{ }^{2}-a^{2}}{2}-2 Q_{i} \alpha_{i}\right. \\
& +2 Q_{i} a+\frac{\alpha_{i}^{2}-a^{2}}{2}+b \alpha_{k}-b a+\frac{\alpha_{k}^{2}-a^{2}}{2}-2 Q_{k} \alpha_{k} \\
& \left.+2 Q_{k} a+\frac{\alpha_{k}-a^{2}}{2}\right] \\
= & \frac{p_{0 i}}{(b-a)^{3}}\left[\frac{b^{2}-a^{2}}{2}+b\left(\alpha_{i}+\alpha_{k}-2 a\right)+\alpha_{i}^{2}-a^{2}\right. \\
& \left.+\alpha_{k}^{2}-a^{2}+2 Q_{i}\left(a-\alpha_{i}\right)+2 Q_{k}\left(a-\alpha_{k}\right)\right]
\end{aligned}
$$

Dependiendo de los valores que tome $\alpha_{j}, j=i, k$, se tienen cuatro posibles casos:

1. $\alpha_{i}=\alpha_{k}=b$,

2. $\alpha_{i}=b, \alpha_{k}=Q_{k}$,

3. $\alpha_{i}=Q_{i}, \alpha_{k}=Q_{k}$,

4. $\alpha_{i}=Q_{i}, \alpha_{k}=b$.

Para el caso 1., la igualdad (B.5) queda de la siguiente manera:

$$
\begin{aligned}
\frac{\partial^{2}}{\partial Q_{i} \partial Q_{k}} E \Pi_{i}\left(Q_{i} \mid Q_{k}\right)= & \frac{p_{0 i}}{(b-a)^{3}}\left[\frac{\left(b^{2}-a^{2}\right)}{2}+b(2 b-2 a)+b^{2}-a^{2}+b^{2}-a^{2}\right. \\
& \left.+2 Q_{i}(a-b)+2 Q_{k}(a-b)\right] \\
= & \frac{p_{0 i}}{(b-a)^{3}}\left[(b-a)\left\{\frac{5}{2}(b+a)+2 b-\left(2 Q_{i}+2 Q_{k}\right)\right\}\right]
\end{aligned}
$$

y (B.6) es no negativa cuando $\frac{9}{4} b+\frac{5}{4} a \geq Q_{i}+Q_{k}$. 
Para el caso 2., la segunda derivada queda de la forma:

$$
\begin{aligned}
\frac{\partial^{2}}{\partial Q_{i} \partial Q_{k}} E \Pi_{i}\left(Q_{i} \mid Q_{k}\right)= & \frac{p_{0 i}}{(b-a)^{3}}\left[\frac{b^{2}-a^{2}}{2}+b\left(b+Q_{k}-2 a\right)+b^{2}-a^{2}\right. \\
& \left.+Q_{k}^{2}-a^{2}+2 Q_{i}(a-b)+2 Q_{k}\left(a-Q_{k}\right)\right] \\
= & \frac{p_{0 i}}{(b-a)^{3}}\left[\frac{5}{2}\left(b^{2}-a^{2}\right)+Q_{k}\left(b-Q_{k}+2 a\right)\right. \\
& \left.+Q_{i}(2 a-2 b)-2 a b\right]
\end{aligned}
$$

se tiene que, la segunda derivada obtenida en (B.7), es no negativa cuando $\frac{5}{2}\left(b^{2}-a^{2}\right)+Q_{k}\left(b-Q_{k}+2 a\right)+2 a \geq 2 a b+2 Q_{i}(b-a)$.

En el caso 3., (B.5) queda:

$$
\begin{aligned}
\frac{\partial^{2}}{\partial Q_{i} \partial Q_{k}} E \Pi_{i}\left(Q_{i} \mid Q_{k}\right)= & \frac{p_{0 i}}{(b-a)^{3}}\left[\frac{b^{2}-a^{2}}{2}+b\left(Q_{i}+Q_{k}-2 a\right)+Q_{i}^{2}-a^{2}\right. \\
& \left.+Q_{k}^{2}-a^{2}+2 Q_{i}\left(a-Q_{i}\right)+2 Q_{k}\left(a-Q_{k}\right)\right] \\
= & \frac{p_{0 i}}{(b-a)^{3}}\left[\frac{b^{2}-a^{2}}{2}+Q_{i}\left(b-Q_{i}+2 a\right)\right. \\
& \left.+Q_{k}\left(b-Q_{k}+2 a\right)-a(2+2 a)\right]
\end{aligned}
$$

(B.8) es no negativa, ya que $2 a Q_{\ell} \geq 2 a^{2} \geq 2 a$, pues $Q_{\ell} \geq a$, para $\ell=i, k$; así $2 a\left(Q_{i}+Q_{k}\right) \geq 2 a(a+1)$, de esto que (B.8) sea no negativa.

Para el caso 4., la expresión (B.5) queda como:

$$
\begin{aligned}
\frac{\partial^{2}}{\partial Q_{i} \partial Q_{k}} E \Pi_{i}\left(Q_{i} \mid Q_{k}\right)= & \frac{p_{0 i}}{(b-a)^{3}}\left[\frac{b^{2}-a^{2}}{2}+b\left(Q_{i}+b-2 a\right)+Q_{i}^{2}-a^{2}\right. \\
& \left.+b^{2}-a^{2}+2 Q_{i}\left(a-Q_{i}\right)+2 Q_{k}(a-b)\right] \\
= & \frac{p_{0 i}}{(b-a)^{3}}\left[\frac{5}{2}\left(b^{2}-a^{2}\right)+Q_{i}\left(b-Q_{i}+2 a\right)\right. \\
& \left.+2 Q_{k}(a-b)-2 a b\right]
\end{aligned}
$$

y cuando $\frac{5}{2}\left(b^{2}-a^{2}\right)+Q_{i}\left(b-Q_{i}+2 a\right) \geq Q_{k}(b-a)+2 a b$, se tiene que (B.9) es no negativa. 
Así, para los casos descritos, la función de utilidad esperada, con demandas uniformes, tiene diferencias crecientes y como las funciones están definidas en subconjuntos de $\mathbb{R}^{n}$, por corolario 1.53 , las funciones son supermodulares. 


\section{Apéndice $\mathrm{C}$}

\section{Códigos}

Listing C.1: Código optimización Round-Robin

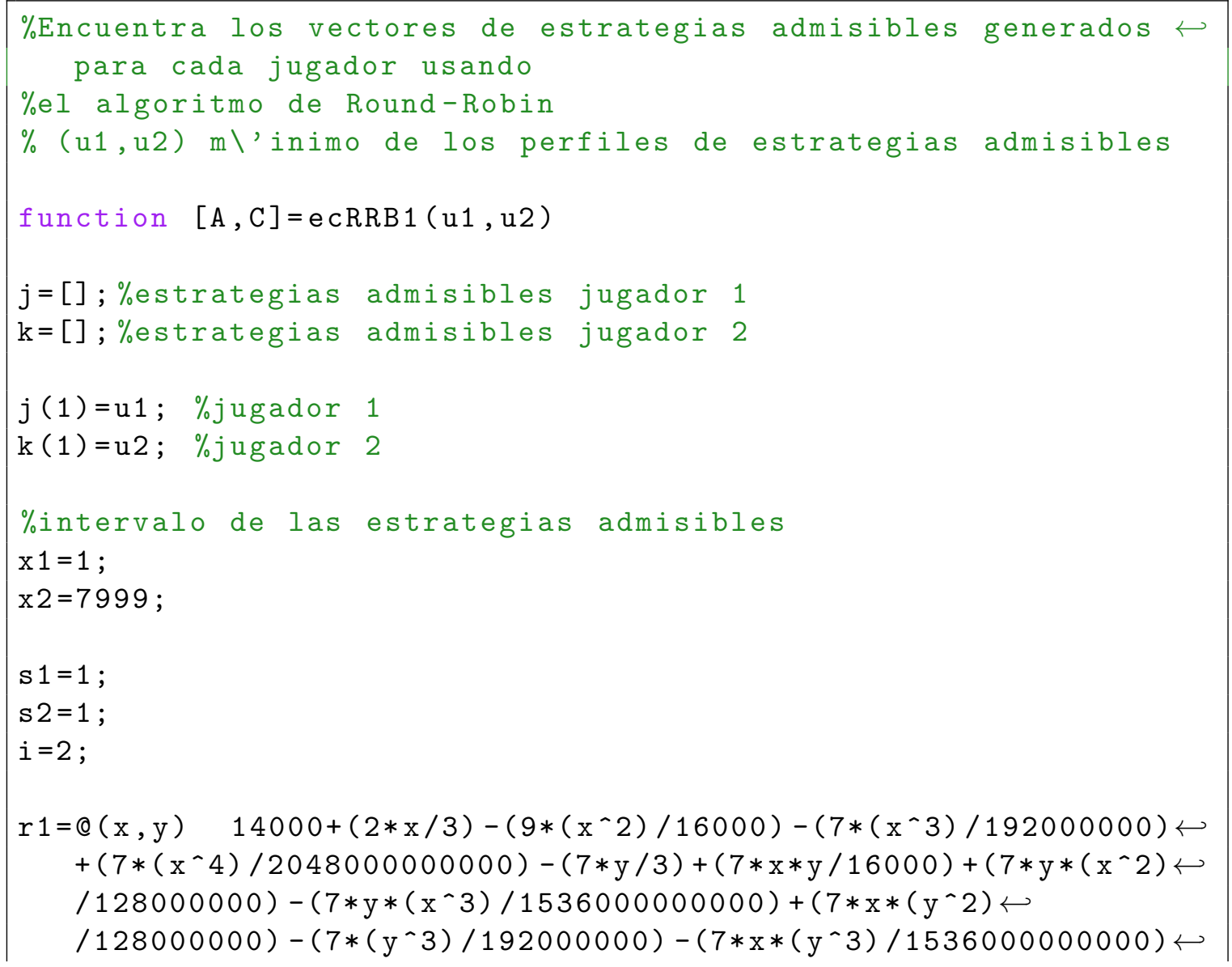


22

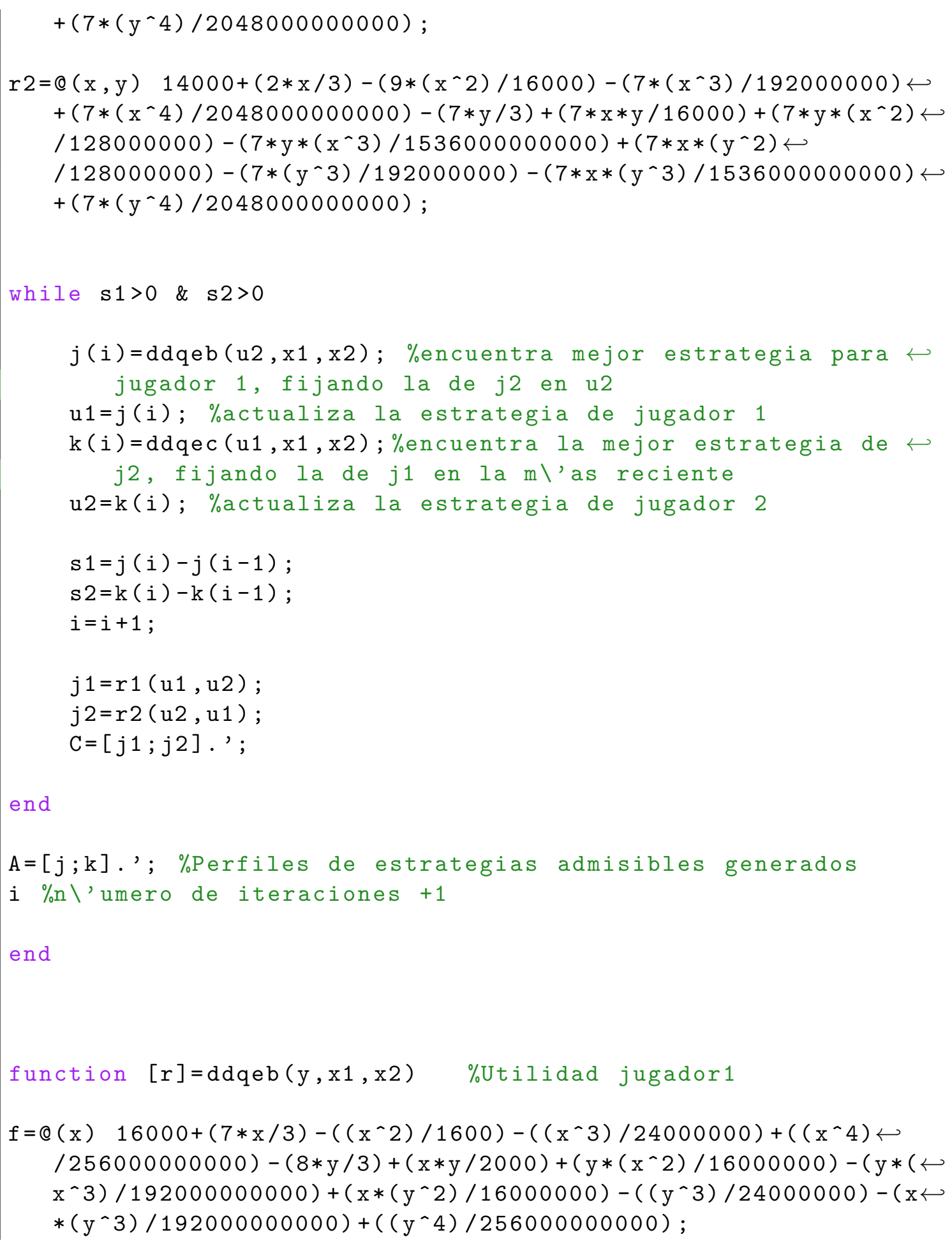




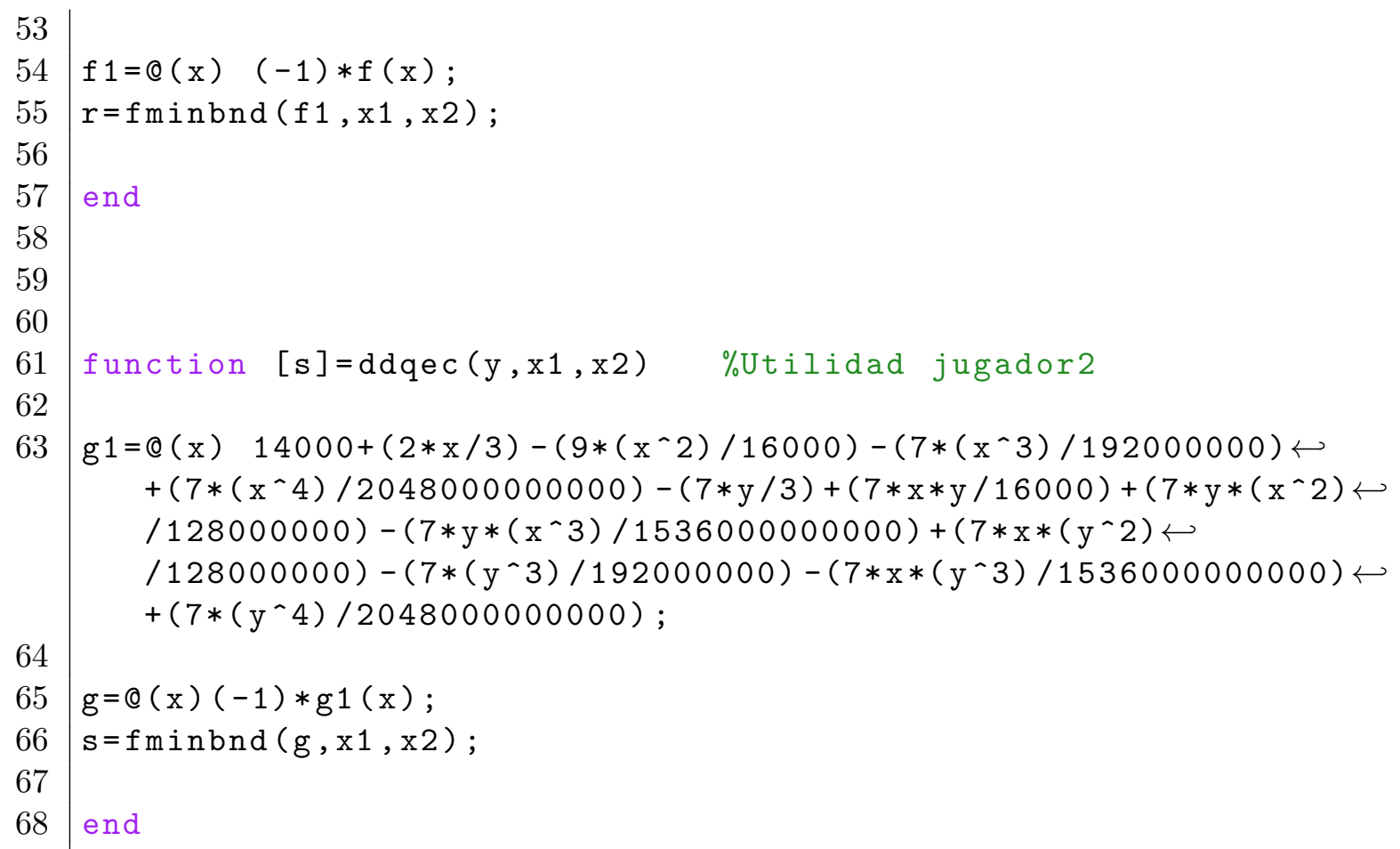


Figura C.1: Código para generar las funciones de utilidad

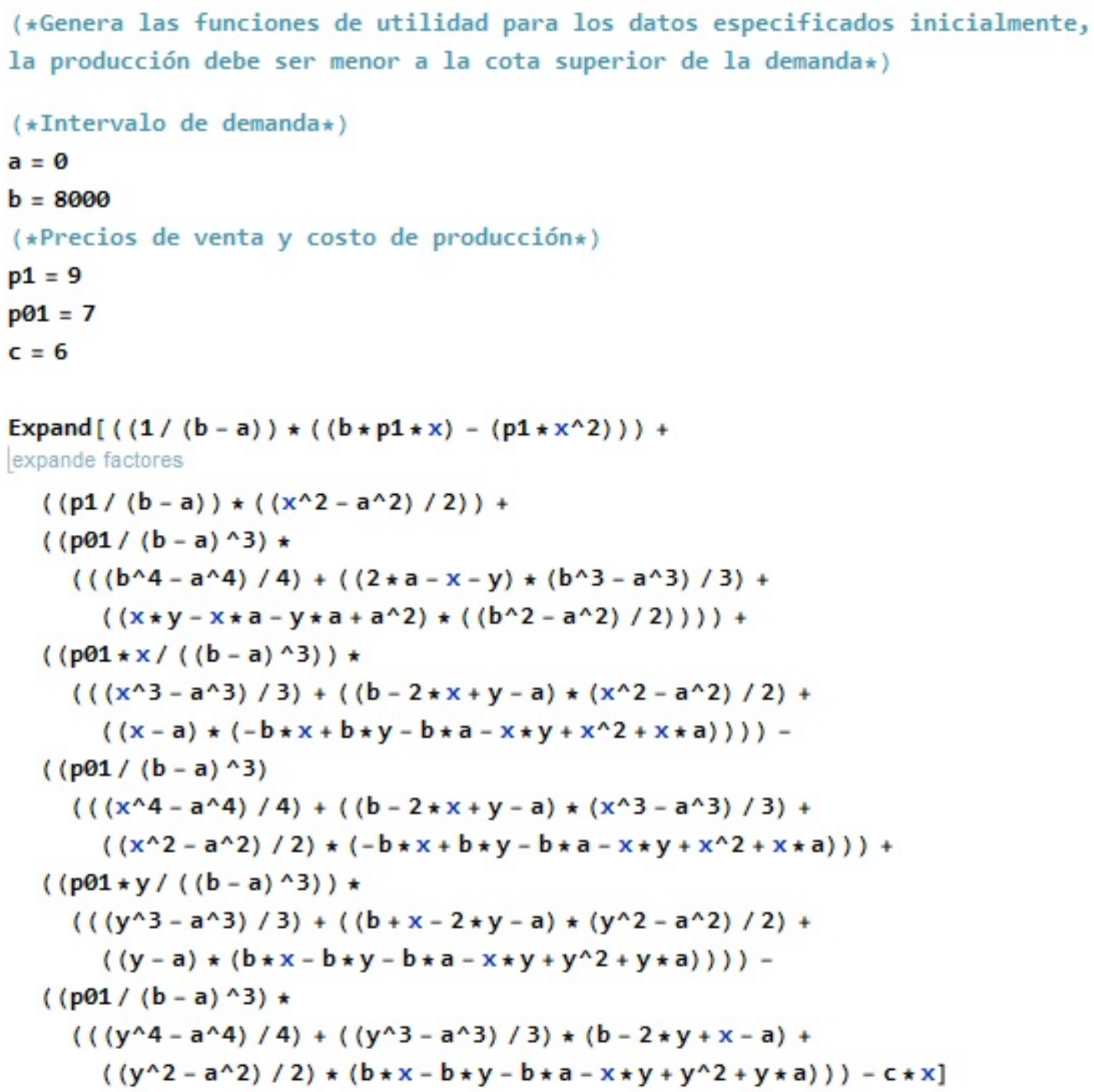




\section{Bibliografía}

[1] Amir, Rabah. Supermodularity and Complementarity in Economics: An Elementary Survey. Southern Economic Journal, Vol. 71, No. 3 (2005), 636-660.

[2] Fang, Xiang. Capacity Games for Partially Complementary Products Under Multivariate Random Demands. Naval Research Logistics, Vol. 59, No. 2 (2012), 146-159.

[3] Fang, X. And Wang, Y. A Model for Partial Product Complementarity and Strategic Production Decisions under Demand Uncertainty. Production and Operations Management, Vol. 19, No. 3 (2010), 322-342.

[4] Flores-Hernández, R.M. And Montes-De-OcA, R. Noncooperative games with non-compact joint strategies sets: increasing optimal responses and approximation to equilibrium points. Kybernetika, Vol. 47, No. 2 (2011), 207-221.

[5] Long, C., Zhang, Q., Li, B., Yang, H. And Guan, X. Non-Cooperative Power Control for Wireless Ad Hoc Networks with Repeated Games. IEEE Journal on Selected Areas in Communications, Vol. 25, No. 6 (2007), 1101-1112.

[6] Nash, John. Non-Cooperative Games. Annals of Mathematics, Second Series, Vol. 54, No. 2 (1951), 286-295.

[7] Sundaram, Rangarajan K. A first course in optimization theory. Cambridge University Press, first edition, 1996.

[8] Taylor, H. M. And Karlin S.. An Introduction to Stochastic Modeling. Academic Press, third edition, 1998.

[9] Topkis, Donald M. Equilibrium points in nonzero-sum n-person submodular games. SIAM J. Control and Optimization, Vol. 17, No. 6 (1979), 773-787. 
[10] Topkis, Donald M. Supermodularity and Complementarity. New Jersey: Princeton University Press, 1998.

[11] Topkis, Donald M. The Structure of Sublattices of the Product of $n$ Lattices. Pacific Journal of Mathematics, Vol. 65, No. 2 (1976), 525-532.

[12] Vives, XAVIER. Nash equilibrium with strategic complementarities. Journal of Mathematical Economics, Vol. 19, No. 3 (1990), 305-321.

[13] YaO, David. S-modular games, with queueing applications. Queueing Systems, Vol. 21, No. 3-4 (1995), 449-475.

[14] Zapata, Paloma. Economía política y otros juegos. Una introducción a los juegos no cooperativos. Facultad de Ciencias, UNAM, primera edición, 2007.

[15] Zeidler, Eberhard. Nonlinear Functional Analysis and its Applications vol. $I V$. New York: Springer-Verlag, 1988. 

ANALISIS $Y$ APLICACIÓN DE JUEGOS NO COOPERATIVOS CON ESTRUCTURA SUPERMODULAR

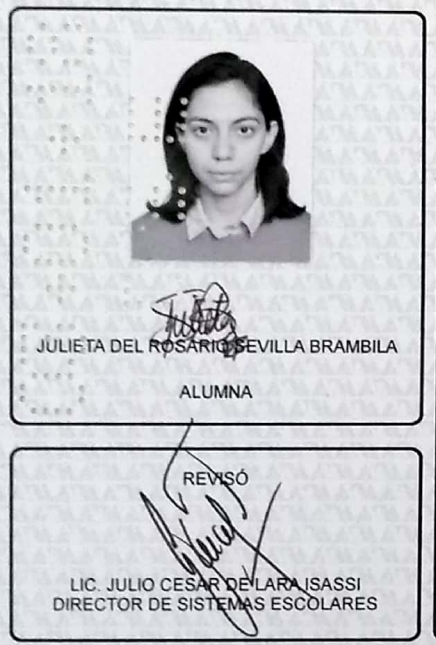

En la Ciudad de México, se presentaron a las 15:00 horas del dí a 13 a de deltapalapa de la Universidad Autol ano Metropolitanidad suscritos miembros del jurado:

DR. JULIO CESAR GARCIA CORTE

DR. VICTOR HUGO VAZOUEZ GUEVARA

DR. JOSE RAUL MONTES DE OCA MACHORRO

Bajo la Presidencia del primero y con carácter de Secretario el ultimo, se reunieron para proceder al Examen de Grado cuya denominación aparece al margen, para la obtención del grado de:

MAESTRA EN CIENCIAS (MATEMÁTICAS APLICADAS E INDUSTRIALES) DE: JULIETA DEL ROSARIO SEVILLA BRAMBILA

$y$ de acuerdo con el artículo 78 fracción III del Reglamento de Estudios Superiores de la Universidad Autónoma Metropolitana, los miembros del jurado resolvieron:

\section{Aprobar}

Acto continuo, el presidente del jurado comunicó a la interesada el resultado de la evaluación $y$, en caso aprobatorio, le fue tomada la protesta.
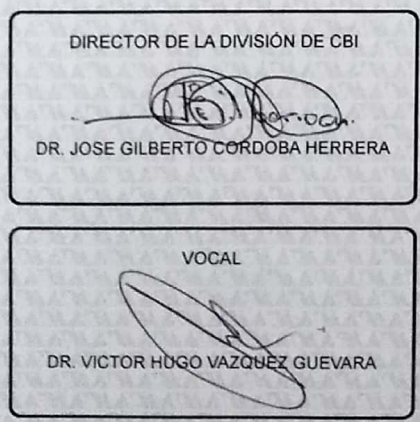

PRESIDENTE

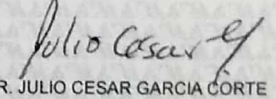

DR. JULIO CESAR GARCIA CORTE

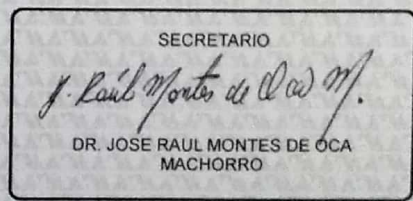

